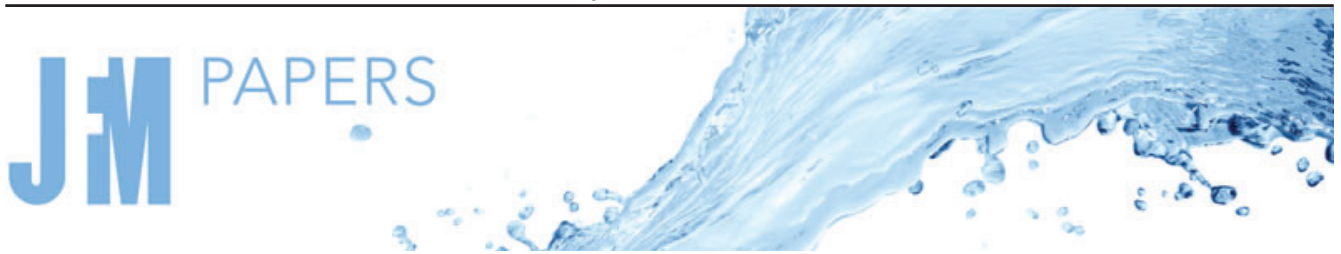

\section{Droplet motion and oscillation on contrasting micro-striated surfaces}

\author{
Hongyu Zhao ${ }^{1}$, Daniel Orejon ${ }^{1}$, Khellil Sefiane ${ }^{1, \dagger}$ and Martin E.R. Shanahan ${ }^{2}$ \\ ${ }^{1}$ School of Engineering, The University of Edinburgh, King's Buildings, Peter Guthrie Tait Road, \\ Edinburgh EH9 3FD, UK \\ ${ }^{2}$ University of Bordeaux, Arts et Metiers ParisTech, I2M, CNRS UMR 5295, F-33400 Talence, France
}

(Received 20 July 2020; revised 2 February 2021; accepted 9 March 2021)

Spontaneous motion of liquid droplets can occur on hydrophobic, micro-structured, solid surfaces comprising a structural gradient. In this study, we examine such motion experimentally and explain our observations by invoking variable droplet-surface interactions (both actuation and resistance forces) arising from the structural gradient. The oscillatory motion of the droplet constitutes an integral aspect of the behaviour and this is incorporated into the overall modelling. The theoretical model features a truncated spheroid for the drop shape (flattened in the region of solid contact) coupled with the oscillatory and alternate leading and trailing motion of the contact line. Results from the model and experiments provide good qualitative and quantitative agreement. The component of the vertical oscillation is found to help overcome wetting hysteresis and actuate the motion, this being a key element for the completeness of the model.

Key words: drops, contact lines, microfluidics

\section{Introduction}

For millimetric droplets and bubbles with dimensions comparable to the capillary length, phenomena such as coalescence (Yeh et al. 2013; Soto et al. 2018), droplet splitting (Chiou, Chang \& Wu 2008), oscillation (Duncombe et al. 2012), bouncing (Reyssat, Pardo \& Quéré 2009) and directional transport (Chaudhury \& Whitesides 1992) under surface gradients are dominated by surface tension forces. Among droplet dynamic actuation and interactions between liquids and solid surfaces, the directional transport of micrometreand millimetre-sized liquid droplets has attracted considerable interest in the past two decades and offered potential benefits in many applications such as chemical analysis and bioassay systems (Velev, Prevo \& Bhatt 2003; Srinivasan, Pamula \& Fair 2004;

$\dagger$ Email address for correspondence: k.sefiane@ed.ac.uk 


\section{H. Zhao, D. Orejon, K. Sefiane and M.E.R. Shanahan}

Wixforth et al. 2004; De Angelis et al. 2011), dropwise condensation heat transfer (Daniel, Chaudhury \& Chen 2001; Macner, Daniel \& Steen 2014), water harvesting (Zheng et al. 2010) and self-cleaning (Blossey 2003).

To control droplet transport on solid surfaces, the interplay and tuning of the different surface tensions involved, i.e. solid-liquid, $\gamma_{S L}$, liquid-vapour, $\gamma$, and solid-vapour, $\gamma_{S V}$, are paramount. One of the common methodologies exploited for droplet manipulation is to create a single sharp contrast (He \& Lee 2003; Kita et al. 2018; Zhao et al. 2020) or a continuous/stepwise gradient (Shastry, Case \& Böhringer 2005, 2006; Yang et al. 2006; Reyssat et al. 2009; Launay et al. 2020) on the solid by adjusting the surface roughness on an intrinsically hydrophobic background, either imposed by the material itself or upon a subsequent coating. In the last century, the influence of roughness on static wetting has been investigated and has been shown to obey the theories of Wenzel (1936) or Cassie \& Baxter (1944) depending on the intrinsic wettability, the structure of the solid surface and the nature of the liquid. On an intrinsically hydrophilic surface, roughness induces a better wetting behaviour because complete penetration of the liquid into the micro-structures occurs, thus causing an increase in the solid-liquid interfacial area (Kim, Moon \& Kim 2016). This is known as the Wenzel state with a consequent enhanced droplet adhesion (Wenzel 1936). On an intrinsically hydrophobic surface, roughness results in a lower wettability as a consequence of the suppression of liquid penetration into the micro-/nano-structures and leads to the presence of air pockets, which effectively reduce the droplet-surface interactions. This is known as the Cassie-Baxter state, in which droplets are more mobile (Cassie \& Baxter 1944). Therefore, introducing a roughness gradient on intrinsically hydrophobic surfaces has been proposed as an effective method to facilitate droplet transport.

With an aim to control droplet motion purely via structural roughness on an intrinsically hydrophobic background, many studies have focused on the design of structured surfaces via micro-fabrication, where the degree of roughness and hence the surface wettability gradient can be precisely adjusted. The first droplet migration reported, owing solely to surface roughness, was achieved on a half-flat-half-rough polydimethylsiloxane (PDMS) substrate, where a droplet placed at the boundary migrated towards the flat area, i.e. the more wettable region, which minimised its surface free energy (He \& Lee 2003). Thereafter, the first continuous surface tension gradient stemming from a progressive change in the solid fraction, i.e. surface roughness, was created by Shastry et al. (2005, 2006). In their work, they proposed a model that accounted for the hysteresis force to be overcome for the migration of the droplet to ensue, which was thereafter validated by experimental observations (Shastry et al. 2006). At the same time, spontaneous droplet motion was achieved on a micro-grooved surface by adjusting the solid fraction of the grooves to impose the necessary driving force for the motion (Yang et al. 2006). By examining hysteresis more closely, it was found that among the surface topography and structural length scale effects, discontinuous segments, such as micro-pillars, influenced hysteresis the most (Öner \& McCarthy 2000). Specifically, more irregular micro-pillar shapes, such as rhombuses or stars, induce higher hysteresis than smoother contoured shapes. Further, the critical angles for the onset of droplet sliding on tilted surfaces were compared among surfaces with different micro-structural shapes, namely micro-pillars and micro-grooves, and for the latter, in both groove and orthogonal directions (Yoshimitsu et al. 2002). The smallest sliding angles, thus lowest hysteresis, were observed for droplet motion on micro-grooved surfaces, i.e. micro-striated surfaces, along the striation direction. 


\section{Droplet motion and oscillation on micro-striated surfaces}

Other works have proposed physical principles relating friction or hysteresis to the micro-structure solid fraction (Xu \& Choi 2012; Kita et al. 2018), and driving force or velocity to the solid fraction gradient (Kita et al. 2018), by considering the droplet-surface interactions and the droplet shape as a linear and continuous gradient between the initial and the final positions. More recently, Zhao et al. (2020) proposed a unified criterion where it is described that the droplet motion, as displacement, and average velocity are solely functions of the structural parameters of the micro-striated surfaces, $D, \bar{v} \propto \Delta \phi / \bar{\phi}$. However, on a superhydrophobic, micro-structured surface, droplets typically do not experience steady motion, especially in the initial stages when they first contact or spread on the surface (Bartolo, Josserand \& Bonn 2005; Yang et al. 2006; Jung \& Bhushan 2008; Reyssat et al. 2009; Wildeman et al. 2016; Kita et al. 2018; Gordillo, Riboux \& Quintero 2019; Launay et al. 2020). Although most works on droplet motion on micro-structured surfaces have reported the presence of an oscillatory behaviour (Yang et al. 2006; Kita et al. 2018; Launay et al. 2020; Zhao et al. 2020) even when the droplet is deposited gently on the surface, the influence of such oscillatory behaviour on the droplet motion has not been further pursued or investigated. The existence of oscillations has an important influence on droplet behaviour and has been used to realise droplet motion even without a wettability gradient/contrast by surface vibration (Brunet, Eggers \& Deegan 2007; Shastry, Taylor \& Bohringer 2007; Noblin, Kofman \& Celestini 2009).

One of the first works on the oscillatory behaviour of (free) droplets controlled by surface tension is attributable to Lord Rayleigh (1879). Oscillations caused by a small distortion from the equilibrium shape were investigated much later in different circumstances such as in large electric fields (Morrison, Leavitt \& Wortman 1981) or in gaseous environments upon break-up from a liquid jet (Becker, Hiller \& Kowalewski 1991). Some theoretical works (Courty, Lagubeau \& Tixier 2006; Chevy et al. 2012) have provided models of the droplet oscillation on non-wetting substrates, where the oscillation period was investigated. To understand the interaction between vertical oscillation and horizontal migration, a determination of the behaviour of the contact line (CL) is essential; however, none of the theoretical works mentioned previously investigated the behaviour of the CL under the influence of oscillations. The simulation work of Lyubimov, Lyubimova \& Shklyaev (2006) investigated free and forced oscillations of a hemispherical droplet on substrates with different conditions of the CL (free or pinned). On hydrophobic, micro-structured surfaces comprising a wettability contrast, the simulation work of Moradi, Varnik \& Steinbach (2010) captured the oscillatory behaviour of the CL. However, no theoretical model to date has yet been proposed to describe the free oscillatory motion of droplets and the CL on micro-structured surfaces. Further, to date, the lack of knowledge of the interactions between the oscillatory motion and the unidirectional migration remains.

The purpose of this work is to propose a more complete, dynamic picture of the motion of a droplet on a hydrophobic surface under the influence of surface wettability contrast, inertia, friction and hysteresis. First, this study will experimentally investigate whether droplets can experience a horizontal migration, a vertical oscillatory motion as well as an alternating leading and trailing motion of the CL. Second, a dynamic physical model will be developed to explain the phenomena observed, where the vertical oscillatory motion and the horizontal migration have been coupled. Subsequently, the model will be compared with the experimental observations. 


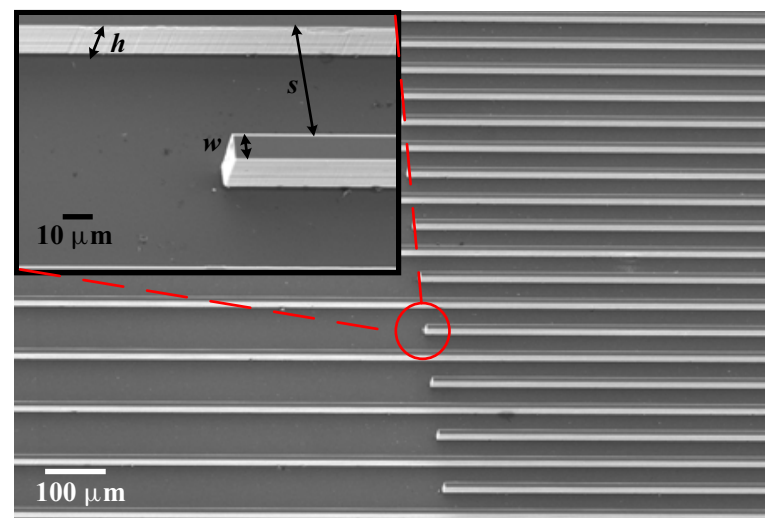

Figure 1. Scanning electron microscopy (SEM) image showing the structure at the boundary of micro-striations on the surface with $w=10 \mu \mathrm{m}, s=90 \mu \mathrm{m}\left(\phi_{L}=0.10\right)$ on the left and $w=10 \mu \mathrm{m}$, $s=40 \mu \mathrm{m}\left(\phi_{R}=0.20\right)$ on the right. Inset shows magnification at the micro-striation boundary. Geometrical parameters $h, w$ and $s$ are included in the inset for clarity.

\section{Materials and methods}

The solid surfaces used to investigate the spontaneous droplet motion across a contrasting wettability boundary were hydrophobic striated micro-structures. It is noted here that we define a boundary as the intersection between the regions of the solid micro-structured surface with different solid fraction parameters, which is different from the conventional definition of interfacial boundary widely used in the fields of hydrodynamics and fluid mechanics. Parallel, geometric striations, of a top-hat profile, were etched via the deep reactive-ion etching (the Deep-RIE or Bosch process) (Laermer \& Schilp 1996) onto smooth silicon wafers with a pre-designed mask, which led to an excellent spatial resolution of the motifs, as may be seen in the micrograph of figure 1. Different levels of wettability were achieved by imposing different geometric parameters, i.e. different solid fractions or, expressed alternatively, different ratios of the top solid surface area to the whole projected surface area, between structural units with a well-defined boundary. All micro-striations were of constant height, $h$, equal to $20 \mu \mathrm{m}$, but with different values of width, $w$, and spacing, $s$. The solids fabricated offered a wide range of solid fractions, $\phi$, defined as $\phi=w /(w+s)$. Surfaces with $\phi$ varying from 0.10 to 0.91 were produced. Following Deep-RIE and further cleaning, the surfaces were coated with a perfluorodecyltrichlorosilane (FDTS) monolayer that imposed homogeneous intrinsic hydrophobicity on the micro-striated structures (see appendix A.1 for fabrication details). Wettability on both micro-striated and flat FDTS-coated surfaces was characterised by measuring the apparent, $\theta_{a p p}$, advancing, $\theta_{a d v}$, and receding, $\theta_{\text {rec }}$, contact angles (CAs). CAs were measured using a Drop Shape Analyser 100 (DSA 100, Krüss GmbH, Hamburg, Germany) and are given in table 1 (see appendix A.2 for detailed information on CA measurements). Droplets of distilled water with a volume of approximately $9 \mu \mathrm{l}$ (corresponding to a droplet radius upon deposition below the capillary length for water, this latter being approximately $2.7 \mathrm{~mm}$ ) were gently deposited at the boundary of two contrasting micro-striated surfaces (see appendix A.2 for detailed information on droplet volume). Experimental observations of the droplet motion were recorded with a high-speed camera (Chronos 1.4, Kron Technologies Inc., Canada) at $1000 \mathrm{fps}$ and subsequently analysed with a custom-made MATLAB ${ }^{\circledR}$ code. 


$\begin{array}{lcccccc}\text { Unit No. } & w(\mu \mathrm{m}) & s(\mu \mathrm{m}) & \phi & \theta_{\text {app }}(\mathrm{deg} .) & \theta_{\text {adv }}(\mathrm{deg} .) & \theta_{\text {rec }}(\mathrm{deg} .) \\ 1 & 10 & 90 & 0.10 & 155 \pm 1 & 158 \pm 1 & 151 \pm 1 \\ 2 & 10 & 40 & 0.20 & 146 \pm 1 & 151 \pm 1 & 143 \pm 1 \\ 3 & 10 & 23 & 0.30 & 139 \pm 1 & 145 \pm 2 & 135 \pm 1 \\ 4 & 10 & 15 & 0.40 & 132 \pm 1 & 140 \pm 1 & 128 \pm 1 \\ 5 & 10 & 10 & 0.50 & 127 \pm 1 & 135 \pm 1 & 122 \pm 1 \\ 6 & 10 & 7 & 0.59 & 123 \pm 1 & 131 \pm 2 & 116 \pm 1 \\ 7 & 10 & 5 & 0.67 & 119 \pm 1 & 129 \pm 1 & 111 \pm 2 \\ 8 & 20 & 5 & 0.80 & 114 \pm 2 & 124 \pm 1 & 106 \pm 1 \\ 9 & 50 & 5 & 0.91 & 110 \pm 1 & 120 \pm 1 & 103 \pm 3 \\ \text { Flat } & - & - & - & 111 \pm 1 & 119 \pm 1 & 105 \pm 3\end{array}$

Table 1. Characteristics of the geometric parameters of the micro-structure, width, $w$, spacing, $s$, and solid fraction, $\phi$, and the contact angles (CAs) of water on each of the surface units. Here $\theta_{a p p}, \theta_{a d v}$ and $\theta_{\text {rec }}$ are the apparent, advancing and receding CAs of water on each surface unit. All CAs were measured in the orthogonal direction to the micro-striations (Zhao et al. 2020).

\section{An example of droplet dynamic motion}

A representative example of droplet motion across the boundary of two contrasting units, specifically, left unit $\left(\phi_{L}=0.10\right)$ and right unit $\left(\phi_{R}=0.20\right)$, is shown in figure 2(a). The dynamic CAs along with the positions of the centre of mass (c.m.) in the horizontal $(x)$ and vertical $(y)$ directions, and the contact points (CPs), which are extracted from the custom-built MATLAB ${ }^{\circledR}$ code, are plotted in figures $2(b)$ and $2(c)$, respectively. CPs are defined as the front (right) and rear (left) points of the CL in the direction of the motion along the micro-striations, as can be seen in the side view in figure 2(a).

After deposition at the contrasting boundary, the droplet undergoes horizontal motion (c.m., $x$ ) towards the right surface unit as a result of the imposed wettability contrast and consequently the unbalanced wetting and dewetting behaviours of the CL as the system attempts to minimise its overall surface free energy. In addition to its horizontal motion, the droplet simultaneously experiences rapid damping of the oscillations occurring in the vertical direction (c.m., y), at an initial oscillation period of approximately $20 \mathrm{~ms}$, as shown in figure 2(c). This oscillatory behaviour, in turn, influences the dynamic CAs as well as the behaviour of the CPs in figures $2(b)$ and 2(c), respectively. Just after deposition, from time $t=0 \mathrm{~ms}$ to $t \sim 10 \mathrm{~ms}$, the droplet spreads slightly over the surface, with left and right CPs moving away from each other. Thereafter, from $t \sim 10 \mathrm{~ms}$ to $t \sim 20 \mathrm{~ms}$, both CPs dewet/recede towards the droplet centre. Photographs taken during this period are shown in figure 2(a). The CPs then continue to oscillate while the droplet migrates in the same direction of the wettability contrast, i.e. towards the right, from $t \sim 20 \mathrm{~ms}$ to $t \sim 100 \mathrm{~ms}$, as represented in figures $2(a)$ and $2(c)$. The variation in solid fraction across the boundary provides a contrast in wettability and therefore a net force towards the higher solid fraction unit side, which induces the droplet to migrate. From figure 2(b), at approximately $60 \mathrm{~ms}$, the sudden drop of the left CA indicates that the droplet has moved completely across the boundary and finds itself on a uniform, homogeneous section of the substrate, where there is no longer any gradient in wettability, as is clearly shown in figures $2(a)$ and $2(c)$. Although no sudden change in the horizontal motion of the c.m. is noticeable after the CL fully moves across the boundary, the driving force induced by the structural gradient now vanishes, and any further droplet motion solely arises from the inertial overshoot until the droplet finally comes to a rest. 
(a)

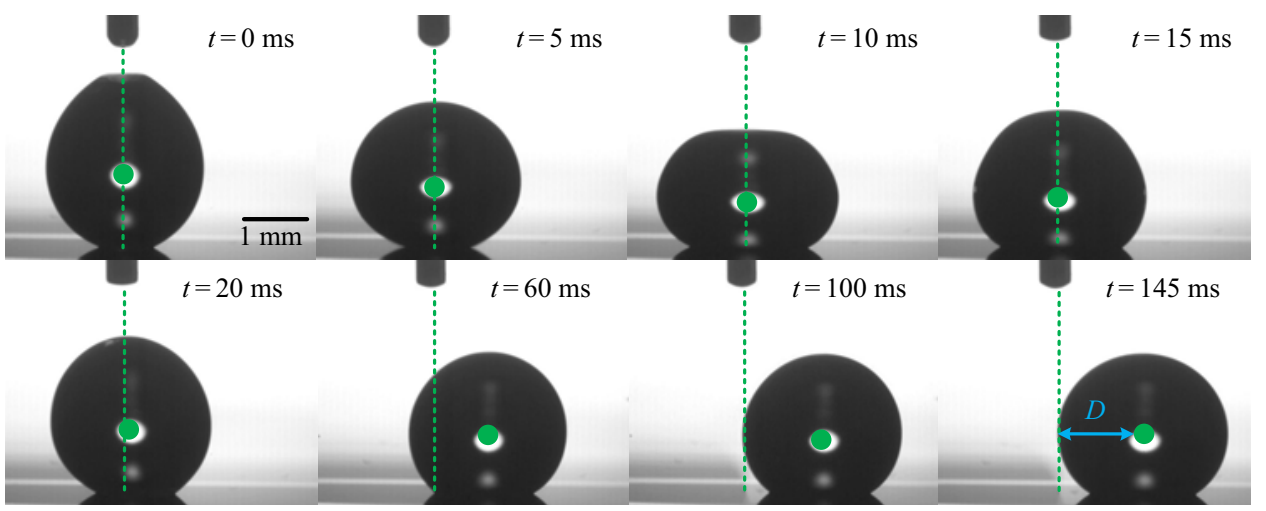

(b)
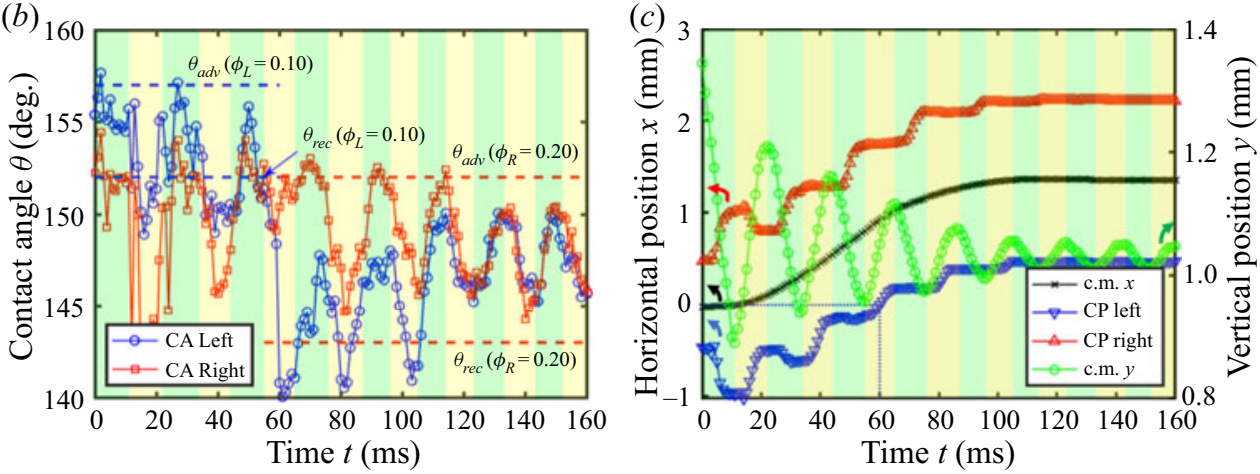

Figure 2. (a) Sequential photographs of a $9 \mu 1$ droplet moving on the boundary of unit $1\left(\phi_{L}=0.10\right)$ and unit $2\left(\phi_{R}=0.20\right)$. Parts of the period of motion $(5-20,60$ and $100 \mathrm{~ms})$ and the final position $(145 \mathrm{~ms})$ with displacement, $D$, of the centre of mass (c.m.) from the initial position to the instantaneous position are presented. The green dots represent the c.m. of the droplet and the horizontal dotted line shows the position of the boundary on the surface, which are clearly visible in the last photo (at $145 \mathrm{~ms}$ ). (b) Right (red) and left (blue) dynamic CAs of the droplet versus time. Note that the sudden drop of the left CA at approximately $60 \mathrm{~ms}$ arises from the completion of the CL motion across the boundary. Experimental values of $\theta_{a d v}$ and $\theta_{\text {rec }}$ for each independent unit from table 1 are indicated by the dotted lines for $\theta_{\text {adv }}=158^{\circ}$ and $\theta_{\text {rec }}=151^{\circ}$ for unit $1\left(\phi_{L}=0.10\right)$, and $\theta_{a d v}=151^{\circ}$ and $\theta_{\text {rec }}=143^{\circ}$ for unit $2\left(\phi_{R}=0.20\right)$. (c) Horizontal motion of left and right contact points (CPs) and c.m., and vertical oscillation of the c.m. with time. Green/yellow shaded stripes marked in $(b, c)$ indicate the downward/upward motion periods of c.m., $y$. Arrows in $(c)$ indicate the corresponding axis for each curve.

Furthermore, when looking closely at figures $2(b)$ and 2(c), the CPs move synchronously with the vertical oscillation. They move only when the dynamic CAs are found beyond the $\theta_{a d v}$ and $\theta_{\text {rec }}$ of the corresponding surfaces that the CPs move on, as indicated by the dashed lines. Otherwise they are pinned owing to hysteresis and insufficient driving force. When the CPs are pinned temporarily (edges of each shaded stripe), the oscillation leads to deformation of the droplet shape near the CPs and, therefore, the consequent rapid changes in the dynamic CAs, until the CPs start to advance/recede again. When the CPs move (middle of each shaded stripe), the dynamic CAs change is less pronounced, when compared with the case of pinned CPs, and their values revolve around the peak/trough values. To determine the interaction between the vertical oscillation and horizontal migration, it is crucial to know how the droplet oscillates and its influence on the CL. 
(a)

(b)

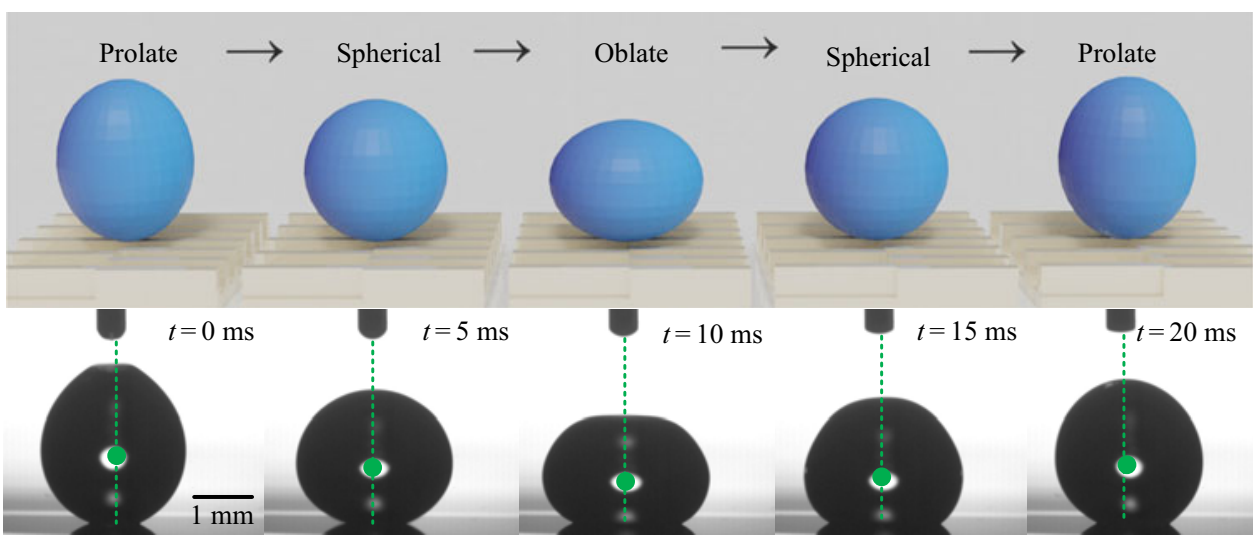

Figure 3. (a) Schematic diagram of droplet evolution in the oscillatory model and (b) corresponding photographs of motion in figure $2(a)$ between $t=0 \mathrm{~ms}$ and $t=20 \mathrm{~ms}$.

\section{Theoretical model}

To explain the dynamics of the oscillatory motion as well as the final position of the droplet with respect to the solid wetting boundary, we have developed and coupled simple mathematical models. Before the droplet leaves the boundary, its motion can be regarded as the superposition of two components: vertical damped oscillation and horizontal migration. As a preliminary step, we model a liquid spheroid to describe the vertical oscillatory motion observed. Subsequently, we refine the full spheroid model to a truncated spheroid, which allows us to connect the vertical motion with the horizontal CL behaviour. The CL behaviour is asymmetric, owing to the different wettability imposed across the boundary, and is further accounted for in the alternating CL slipping model. Finally, a horizontal migration model that takes into account the vertical oscillation, friction and CL hysteresis is presented to explain the droplet motion even after it has moved completely across the wettability boundary.

\subsection{Vertical oscillatory model}

From figure 2(a), at deposition $t=0 \mathrm{~ms}$, the shape of the droplet deviates slightly from sphericity. Because the most energetically favourable shape for a liquid droplet (in the absence of gravity) is that of a sphere (or spherical cap, when in contact with a solid), any deviation/deformation from sphericity will increase its overall free energy. The energy increase associated with deformation causes the tendency to return to spherical symmetry, but inertia leads to an overshoot and an oscillatory cycle is established. Consider the left-hand sketch of figure 3(a), where a prolate spheroid of volume $V_{s}$ is a reasonable approximation to the deformed drop at this stage. The radius, $a_{s}$, of the equivalent spherical droplet is given by

$$
a_{s}=\sqrt[3]{\frac{3 V_{s}}{4 \pi}} .
$$

During vertical oscillation, to a reasonable approximation, the droplet modelled by a spheroid changes its shape from prolate $(\varepsilon>0)$ to oblate $(\varepsilon<0)$ with an alternate positive to negative perturbation in the vertical position of the c.m., $\varepsilon$. Therefore, the polar radius of the prolate (subscript $p$ ) or oblate (subscript $o$ ) is $a_{p, o}=a_{s}+\varepsilon$. The time-dependent 


\section{H. Zhao, D. Orejon, K. Sefiane and M.E.R. Shanahan}

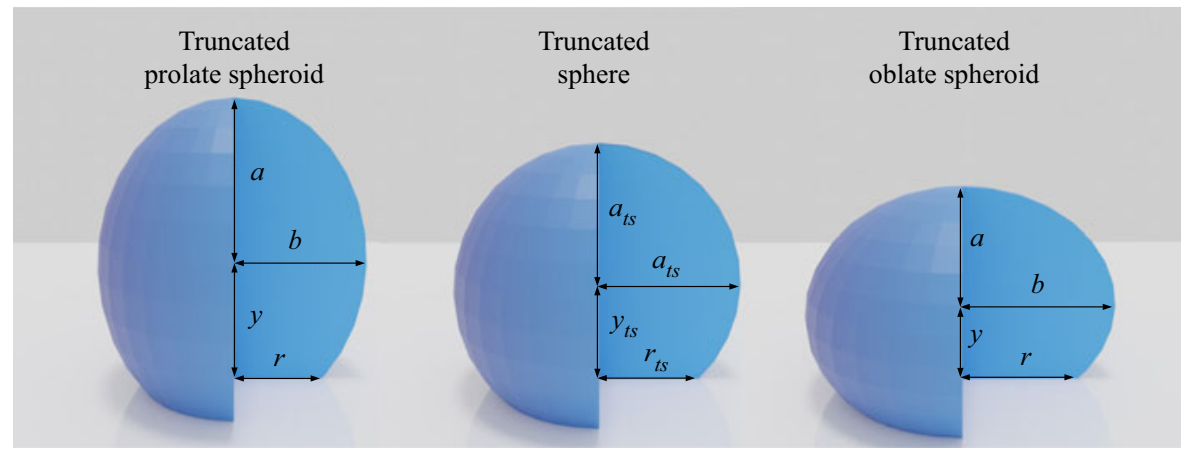

Figure 4. Truncated spheroids, representing a liquid drop, at three different stages: prolate spheroid (left), sphere (middle) and oblate spheroid (right).

polar radius, $a$, i.e. the vertical position of the c.m. of the spheroid, can be then expressed as (see detailed derivation in Appendix B):

$$
a=a_{s}+\varepsilon(t)=a_{s}+A \exp \left(-\frac{H t}{2 m}\right) \cos \left(\sqrt{\frac{16 \pi \gamma}{5 m}-\frac{H^{2}}{4 m^{2}}} \cdot t+\beta\right) .
$$

Here $A$ is the amplitude of the oscillation, $H$ is a dissipation coefficient, $m$ is the mass of the droplet, $\gamma$ is the surface tension of the water-air interface and $\beta$ is the phase angle at $t=0$. The initial condition when the droplet is released from the needle is $\beta=0$. Equation (4.2) also includes the oscillation period, $\tau$ :

$$
\tau=2 \pi\left(\frac{16 \pi \gamma}{5 m}-\frac{H^{2}}{4 m^{2}}\right)^{-1 / 2} .
$$

Clearly a full spheroid would only contact the solid substrate, a plane, at one point. To incorporate a CL of finite length into the model, a truncated spheroidal shape must be used. Consider a truncated sphere of radius of curvature, $a_{t s}$, contact radius, $r_{t s}$, and distance from the centre to the truncation plane, $y_{t s}$, as in figure 4 middle.

By assuming a constant volume condition and $a_{t s}=a_{s}$ for the truncated spheroid during shape change, the relation between the whole and the truncated spheroid can be derived (see detailed derivation of (C5) in Appendix C):

$$
y=y_{t s}\left(a_{t s}+\varepsilon\right) / a_{t s},
$$

where $y$ is the vertical height above the solid of the centre of the truncated prolate spheroid $(\varepsilon>0)$, sphere $\left(\varepsilon=0, y=y_{t s}\right)$ or oblate spheroid $(\varepsilon<0)$ and $\varepsilon$ is derived in (4.2). The base radius, $r$, can be written as (see detailed derivation of (C6) in Appendix C):

$$
r \approx r_{t s}\left(1-\frac{\varepsilon}{2 a_{t s}}\right)
$$

which allows us to follow the change of $r$ with $\varepsilon$. The value of $r$ is not constant during the oscillation. In addition, when the droplet contacts the solid surface, to further account for the influence of hysteresis during the oscillation, we have $y(t)$ (see detailed derivation of 
(C8) in Appendix C):

$$
y(t)=y_{t s}+A^{\prime} \exp \left(-\frac{H t}{2 m}\right) \cos \left(\sqrt{\frac{16 \pi \gamma}{5 m}(1+h)-\frac{H^{2}}{4 m^{2}}} \cdot t+\beta\right),
$$

where $A^{\prime}=A y_{t s} / a_{t s}$ and $h$ is a hysteresis coefficient (the details of its expression can be found in Appendix C).

However, with these models, we still cannot describe the asymmetrical behaviour of the left (subscript $L$ ) and right (subscript $R$ ) parts of the CL, henceforth denoted as $C L_{L}$ and $C L_{R}$, respectively. The hydrophobicity and hysteresis are different for the two units across the boundary, so when the droplet oscillates vertically on the boundary, $C L_{L}$ and $C L_{R}$ experience different forces, which accounts for the horizontal migration of the droplet. We must therefore develop an alternating CL slipping model to account for the different forces at $C L_{L}$ and $C L_{R}$ and relate the horizontal migration of the droplet to the driving force and resistance from the micro-structure units across the boundary.

\subsection{Alternating CL slipping model}

Slippage of the CL is an essential ingredient in our description of droplet bouncing and migration across the wetting boundary. However, the mathematics in three dimensions becomes intractable and therefore we present a simplified two-dimensional model here, which adopts the assumption of a drop of unit thickness, as shown in the inset of figure 6 . Before the droplet finally leaves the boundary entirely (after approximately $60 \mathrm{~ms}$ ), the left and right CAs increase and decrease synchronously, although the difference between their values is typically only a few degrees, as shown in figure $2(b)$. We take $\theta$ to be the dynamic CA, at a given instant, for both $C L_{L}$ and $C L_{R}$. The apparent equilibrium CAs on respectively the left and the right unit surfaces alone are denoted as $\theta_{L}^{*}$ and $\theta_{R}^{*}$. These apparent CAs differ from those on an ideal (flat), solid surface of the same chemical constitution, i.e. from the Young's CA, $\theta_{Y}$ (Young 1805), which follows the Cassie-Baxter relation (Cassie \& Baxter 1944):

$$
\cos \theta_{L, R}^{*}=\phi_{L, R}\left(1+\cos \theta_{Y}\right)-1 .
$$

Adopting in the nomenclature $F_{L}$ and $F_{R}$ to represent the forces acting at $C L_{L}$ and $C L_{R}$ (see the inset of figure 6):

$$
\begin{gathered}
F_{L}=-\phi_{L}\left(\gamma_{S V}-\gamma_{S L}\right)_{L}+\gamma\left(1-\phi_{L}\right)+\gamma \cos \theta=-\gamma\left(\cos \theta_{L}^{*}-\cos \theta\right), \\
F_{R}=\phi_{R}\left(\gamma_{S V}-\gamma_{S L}\right)_{R}+\gamma\left(1-\phi_{L}\right)-\gamma \cos \theta=\gamma\left(\cos \theta_{R}^{*}-\cos \theta\right) .
\end{gathered}
$$

We consider first the case of the c.m. moving in the downwards direction (green stripes, e.g. between $t=0 \mathrm{~ms}$ and $t=10 \mathrm{~ms}$ in figure $2 b, c)$. It is quite plausible that for some time $\theta>\theta_{L}^{*}>\theta_{R}^{*}$, hence $\cos \theta<\cos \theta_{L}^{*}<\cos \theta_{R}^{*}$, and it follows that $\left|F_{L}\right|<\left|F_{R}\right|$. The time-dependent apparent CA, $\theta(t)$, can then be expressed as

$$
\theta(t)=\theta_{L}^{*}+\delta(t)=\theta_{R}^{*}+\delta(t)+\xi
$$

where $\xi$ is a small, positive constant $\left(\xi=\theta_{L}^{*}-\theta_{R}^{*}\right.$, the intrinsic difference between $\theta_{L}^{*}$ and $\theta_{R}^{*}$ ) and $\delta(t)$ is small and a function of time, $t$. The forces acting at $C L_{L}$ and $C L_{R}$ can be 


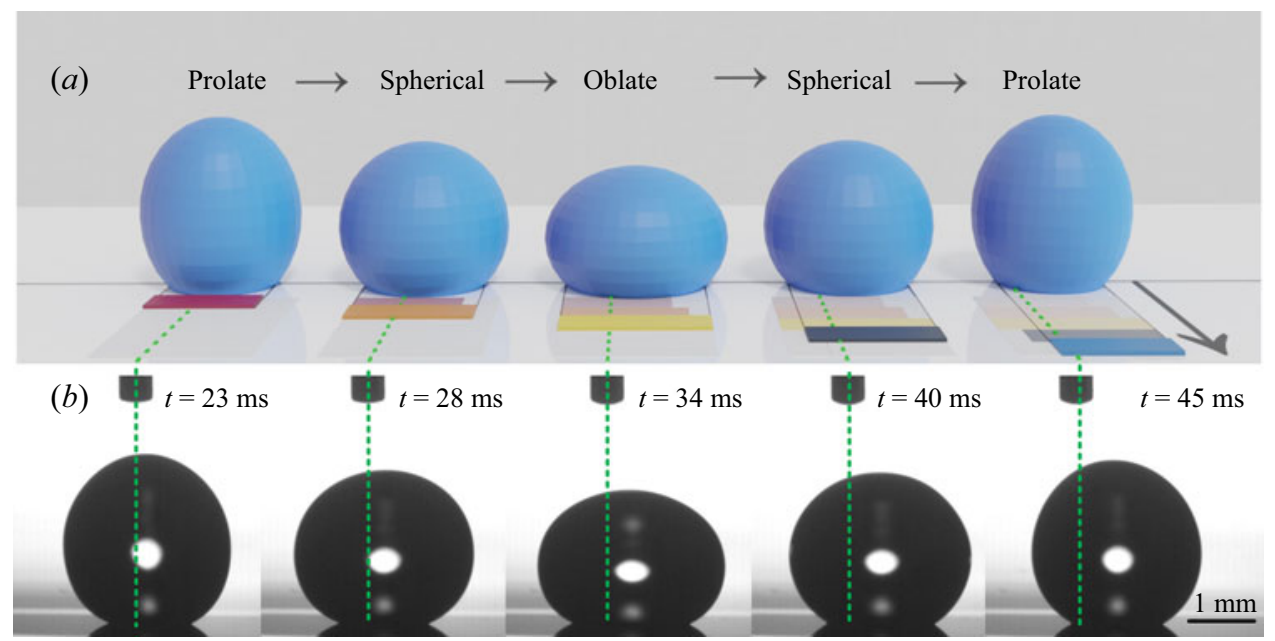

Figure 5. (a) Schematic diagram of evolution of droplet shape and position in the oscillatory truncated model. Colour bars show the length and position of footprints in different states. (b) Sequential photographs of a $9 \mu 1$ droplet moving on the boundary of unit $1\left(\phi_{L}=0.10\right)$ and unit $2\left(\phi_{R}=0.20\right)$ for comparison. Dotted lines indicate the position of the contrast boundary.

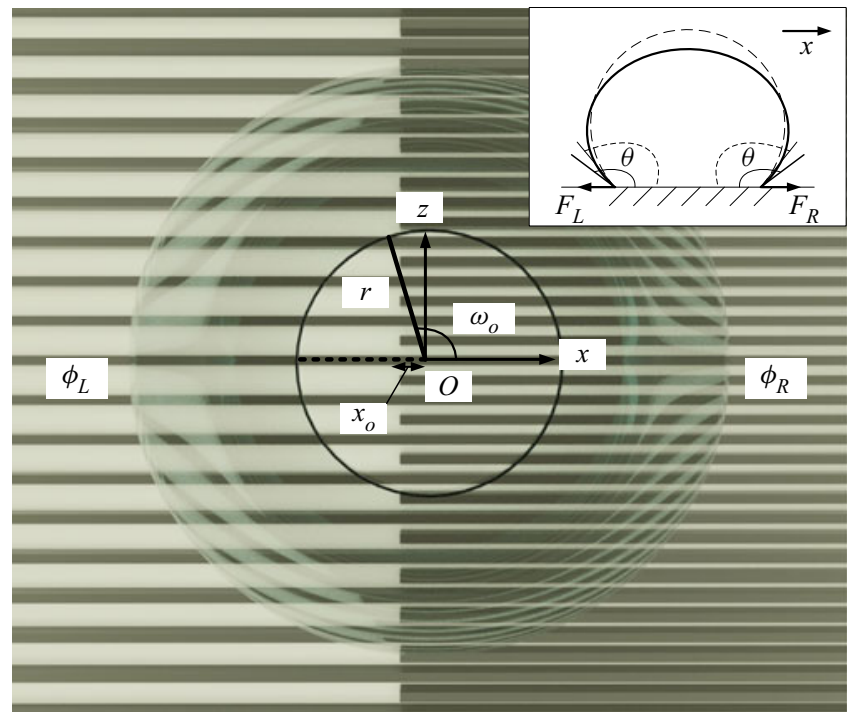

Figure 6. Schematic diagram of the droplet footprint across the boundary of the wettability contrast with a displacement $x_{o}$. The origin, $O$, is set to be the centre of the footprint contacted with the solid surface, which moves with the droplet. Inset shows the directions of the surface forces acting on the $C L_{L}$ and $C L_{R}$.

written as

$$
\begin{gathered}
F_{L}(t) \approx-\gamma \sin \theta_{L}^{*} \cdot \delta(t) \\
F_{R}(t) \approx \gamma \sin \theta_{R}^{*} \cdot(\xi+\delta(t)) .
\end{gathered}
$$

Note that $\delta(t)$ is positive at this stage. There will be some resistance to motion of the CLs, $\Psi_{L}$ and $\Psi_{R}$ for the left and right surface, respectively, which may be interpreted as 


\section{Droplet motion and oscillation on micro-striated surfaces}

wetting hysteresis. If $\delta(t)$ is sufficiently large to satisfy the condition:

$$
\Psi_{L}<\left|\gamma \sin \theta_{L}^{*} \cdot \delta(t)\right|, \Psi_{R}<\left|\gamma \sin \theta_{R}^{*} \cdot(\xi+\delta(t))\right|,
$$

then both $C L_{L}$ and $C L_{R}$ move. This corresponds to the situation observed just following droplet deposition. Thereafter, as damping of the oscillations takes place (between $t \sim 30$ $\mathrm{ms}$ and $t \sim 60 \mathrm{~ms}$ in figure $2 b, c)$, there is a period when

$$
\left|\gamma \sin \theta_{L}^{*} \cdot \delta(t)\right|<\Psi_{L}, \Psi_{R}<\left|\gamma \sin \theta_{R}^{*} \cdot(\xi+\delta(t))\right|,
$$

and $C L_{L}$ is anchored by hysteresis whereas $C L_{R}$ slips/advances to the right under a force of ||$\gamma \sin \theta_{R}^{*} *(\xi+\delta(t))\left|-\Psi_{R}\right|$, which results in slight movement of the c.m. to the right, as shown in figure 5a2,a3). As the droplet oscillation loses amplitude, for $t>100 \mathrm{~ms}$ in figure $2(b), \delta(t)$ decreases and we attain the state where

$$
\left|\gamma \sin \theta_{R}^{*} \cdot(\xi+\delta(t))\right|<\Psi_{R},
$$

whereby both $C L_{L}$ (now moves across the boundary) and $C L_{R}$ become anchored (permanently), i.e.the motion comes to an end. For $C L_{L}$ in the non-overshooting case, $\left|\gamma \sin \theta_{L}^{*} \cdot \delta(t)\right|<\Psi_{L}$.

We now consider the upward phase of oscillation, when $\delta(t)$ decreases (yellow stripes, e.g. between $t \sim 10 \mathrm{~ms}$ and $t \sim 20 \mathrm{~ms}$ in figure $2 b, c)$. This implies that $\theta_{L}^{*}>\theta_{R}^{*}>\theta$, and we have $\left|F_{L}(t)\right|>\left|F_{R}(t)\right|$ (provided $\xi$ is sufficiently small). Then, (4.11) and (4.12) remain valid but note that $\delta(t)$ is now negative, which implies that $F_{L}(t)$ acts towards the right. Thereafter, there is a period (e.g. between $t \sim 30 \mathrm{~ms}$ and $t \sim 60 \mathrm{~ms}$ in figure $2 b, c$ ) when

$$
\Psi_{L}<\left|\gamma \sin \theta_{L}^{*} \cdot \delta(t)\right|,\left|\gamma \sin \theta_{R}^{*} \cdot(\xi+\delta(t))\right|<\Psi_{R},
$$

for which $C L_{R}$ is anchored whereas $C L_{L}$ slips towards the right (as shown in figure 5a4,a5) under the force ||$\gamma \sin \theta_{L}^{*} * \delta(t)\left|-\Psi_{L}\right|$. As the droplet contacts the structured solid surface, the CLs move synchronously during the upward and downward oscillatory phases and the tendency for the c.m. of the droplet to move to the right-hand side is weak. However, at the later stages, when damping has become significant, in both cases, $\left|F_{L}(t)\right|<\Psi_{L}$ and $\left|F_{R}(t)\right|<\Psi_{R}$, both $C L_{L}$ and $C L_{R}$ are pinned, and the droplet stops moving. Between the onset and the final damping of the droplet motion, there is a stage when $C L_{L}$ and $C L_{R}$ move alternately towards the right, so the main displacement of the c.m. takes place between $t \sim 30 \mathrm{~ms}$ and $t \sim 60 \mathrm{~ms}$ in figure 2(b) and 2(c). In addition, after the droplet has moved totally across the boundary and before it stops (between $t \sim 60 \mathrm{~ms}$ and $t \sim 100 \mathrm{~ms}$ in figure $2(b)$ and $2(c)$, there is a stage when the droplet overshoots for a further distance beyond the boundary. During this stage, the inertia of the c.m. of the droplet is sufficient to pull $C L_{L}$ and to push $C L_{R}$ so that the behaviours of both $C L_{L}$ and $C L_{R}$ remain virtually unchanged as the droplet slides over the boundary until the droplet comes to a rest at the last stage of the migration.

\subsection{Horizontal migration model}

Once the droplet has left the boundary, it cannot move indefinitely because the droplet footprint is completely on a homogeneous micro-striated unit, i.e. in the absence of a wettability contrast, and therefore in the absence of a driving force. Motion is here then limited to that arising from inertia. (Momentum was neglected in the preceding argument, owing to it being of secondary importance, but once the CLs become symmetrical, on the same solid surface texture, this becomes the overriding effect.) Returning to the 


\section{H. Zhao, D. Orejon, K. Sefiane and M.E.R. Shanahan}

more realistic three-dimensional model, the net surface force acting on the droplet sitting on the boundary in the $x$ direction with the centre position at $x_{o}$ (as shown in figure 6) is

$$
\begin{gathered}
F_{d}=2\left|\int_{\omega_{o}}^{\pi} \gamma\left(\cos \theta_{R}^{*}-\cos \theta_{L}^{*}\right) \cos \omega r d \omega\right|=2 \gamma r \sin \omega_{o} \Delta \cos \theta^{*} \\
=2 r \sqrt{1-\left(x_{o} / r\right)^{2}} \gamma \Delta \cos \theta^{*},
\end{gathered}
$$

where $\Delta \cos \theta^{*}=\cos \theta_{R}^{*}-\cos \theta_{L}^{*}, r$ is the droplet contact radius and $\omega_{o}$ is the azimuthal angle corresponding to half of the droplet footprint arc on the right-hand side of the boundary, equal to $\omega_{o}=\pi-\cos ^{-1} x_{o} / r$. We assume that the resistance to motion from the surface, $f_{L, R}$, is directly proportional to the solid/liquid contact area (the assumption of $f_{L, R} \propto R^{2}$ in the present case is reasonable as the comparison of the calculated velocity by the model with experimental results provides a better agreement than for the case where the resistance is dominated by the $\mathrm{CL}, f_{L, R} \propto R$ ):

$$
\begin{array}{r}
f_{L}=2\left|\int_{-r}^{-x_{o}} B \phi_{L} \sqrt{r^{2}-x^{2}} \mathrm{~d} x\right|=2 \gamma B^{*} \phi_{L} r \int_{x_{o} / r}^{1} \sqrt{1-(x / r)^{2}} d(x / r), x \leq r, \\
f_{R}=2\left|\int_{-x_{o}}^{r} B \phi_{R} \sqrt{r^{2}-x^{2}} \mathrm{~d} x\right|=\gamma B^{*} \phi_{R} r\left(\pi-2 \int_{x_{o} / r}^{1} \sqrt{1-(x / r)^{2}} d(x / r)\right), x \leq r,
\end{array}
$$

where $B$ is a coefficient accounting for friction (assumed to be independent of the solid surface motif), $B^{*}$ is normalised $B$ by surface tension, $\gamma$, and droplet contact radius, $r$, and equals $B^{*}=B r / \gamma$. After moving across the boundary, i.e. when $x_{o}>r$, we have $F_{d}=0$, $f_{L}=0$ and

$$
f_{R}=\gamma \pi r B^{*} \phi_{R}, x>r .
$$

To obtain information about the speed, the work done by the forces from (4.17)-(4.20) needs to be calculated. Assuming the normalised displacement of the c.m., $x$, by the droplet contact radius, $r, x^{*}=x / r<1$, and integrating the forces from $x^{*}=0$ to $x^{*}=x_{o}^{*}=x_{o} / r$, the work done by the above-mentioned driving force and left and right friction forces are $W_{F_{d}}\left(x_{o}^{*}\right), W_{f_{L}}\left(x_{o}^{*}\right)$ and $W_{f_{R}}\left(x_{o}^{*}\right)$, respectively, which can be derived as $\left(x_{o}^{*}<1\right)$

$$
\begin{gathered}
W_{F_{d}}\left(x_{o}^{*}\right)=\int_{0}^{x_{o}} F_{d} \mathrm{~d} x=2 \gamma r^{2} \Delta \cos \theta^{*} \int_{0}^{x_{o}^{*}} \sqrt{1-\left(x^{*}\right)^{2}} \mathrm{~d} x^{*}, \\
W_{f_{L}}\left(x_{o}^{*}\right)=\int_{0}^{x_{o}} f_{L} \mathrm{~d} x=r \int_{0}^{x_{o}^{*}} f_{L} \mathrm{~d} x^{*}, \\
W_{f_{R}}\left(x_{o}^{*}\right)=r \int_{0}^{x_{o}^{*}} f_{R} \mathrm{~d} x^{*} .
\end{gathered}
$$

When $x_{o}^{*}>1$, the works become $W_{F_{d}}\left(x_{o}^{*}\right)=W_{F_{d}}(1), W_{f_{L}}\left(x_{o}^{*}\right)=W_{f_{L}}(1)$ and

$$
W_{f_{R}}\left(x_{o}^{*}\right)=W_{f_{R}}(1)+\gamma B^{*} \phi_{R} \pi r^{2}\left(x_{o}^{*}-1\right) .
$$

The contributions from the initial deposition and CL pinning to the kinetic energy must also be taken into account. The vertical oscillation and the droplet horizontal shift are related in the alternating CL slipping model. Further vertical oscillation helps the 


\section{Droplet motion and oscillation on micro-striated surfaces}

droplet to overcome hysteresis, therefore, leading to a larger horizontal kinetic energy. Furthermore, experiments demonstrated that for cases with large hysteresis, at the end of the motion, the droplet wobbled horizontally, with its CL pinning onto the surface before becoming still. This suggests that the inertia force cannot overcome the pinning of the CL and part of the kinetic energy is dissipated without enabling the droplet to move further. In consideration of all the above-mentioned effects, the kinetic energy, $E$, involved in the droplet migration on a striated surface comprising a structural wetting gradient at a given normalised displacement from the boundary, $x_{o}^{*}$, is given by

$$
E\left(x_{o}^{*}\right)=W_{D}\left(x_{o}^{*}\right)+W_{F_{d}}\left(x_{o}^{*}\right)-W_{f_{L}}\left(x_{o}^{*}\right)-W_{f_{R}}\left(x_{o}^{*}\right)-W_{H}\left(x_{o}^{*}\right)=\frac{1}{2} m v^{2}, x_{o}^{*}<1,
$$

where $v$ is the droplet velocity and $W_{D}\left(x_{o}^{*}\right)$ is the net kinetic energy converted from a vertical oscillation. The oscillation results in a forced wetting/dewetting behaviour of the $\mathrm{CL}$, which is asymmetric along the motion direction, and contributes to the value of $E$ even without a wettability gradient (Dong, Holmes \& Böhringer 2017). In the present case, the asymmetry of the wetting/dewetting behaviour is solely caused by the surface morphology, which vanishes once the droplet moves across the boundary, and $W_{D}\left(x_{o}^{*}\right)$ does not contribute further to the kinetic energy. Here $W_{H}\left(x_{o}^{*}\right)$ is the kinetic energy dissipated after the $\mathrm{CL}$ is pinned and before the droplet comes to a final halt. Owing to the existence of hysteresis, the CL stops moving prior to the droplet, and the droplet starts 'wobbling' under the 'extra' kinetic energy, $W_{H}\left(x_{o}^{*}\right)$, which has no contribution to the droplet motion. For the overshoot cases, $E(1)>0$, which means when the whole footprint leaves the boundary, the kinetic energy of the droplet is non-zero, which drives the droplet to move further from the boundary. For non-overshoot cases, $E\left(x_{m}^{*}\right)=0$, where the maximum normalised displacement, $x_{m}^{*}<1$, which means the kinetic energy dissipates totally before the droplet leaves the boundary.

According to (B7) in Appendix B, $W_{D}\left(x_{o}^{*}\right)$ should be a function of the change in potential energy, upon gentle deposition, from the initial deposition state to the equilibrium state, as $W_{D}\left(x_{o}^{*}\right) \propto 8 \pi \gamma \varepsilon_{m}^{2} / 5$, where $\varepsilon_{m}$ is the maximum position change of the c.m., $y$ and $\varepsilon_{m} \propto a_{t s}-y_{t s}$. Moreover, the kinetic energy converted from the vertical oscillation contributes to both sides of the CL motion; therefore, the increased driving force in the direction of the droplet motion should be a function of the difference in the projected length of the CL in the perpendicular direction in a similar fashion to (4.17) and (4.21). Then, $W_{D}\left(x_{o}^{*}\right)$ is expressed as

$$
W_{D}\left(x_{o}^{*}\right)=C \frac{8 \pi \gamma\left(a_{t s}-y_{t s}\right)^{2}}{5} \int_{0}^{x_{o}^{*}} \sqrt{1-\left(x^{*}\right)^{2}} \mathrm{~d} x^{*},
$$

where $C$ is a factor accounting for the proportion of the energy converted from the total vertical mechanical energy by the droplet shape oscillation.

The hysteresis term, $W_{H}\left(x_{o}^{*}\right)$, appears only when the motion comes to its end. From the experimental results, we found a continuity of the kinetic energy declining to zero instead of a sudden drop when the CL was finally pinned. In addition, in some cases, we were even able to see a slight horizontal wobbling of the c.m. when CL was pinned. We also noticed that on the surface with higher $\bar{\phi}$, CL was pinned more easily and the velocity declined at a faster rate. To take into account this part of the energy and satisfy the kinetic energy continuity, we assume

$$
W_{H}\left(x_{o}^{*}\right) \propto Z\left(\frac{x_{o}^{*}}{x_{m}^{*}}\right)^{k},
$$




\section{H. Zhao, D. Orejon, K. Sefiane and M.E.R. Shanahan}

where $k$ is a factor indicating how fast $W_{H}\left(x_{o}^{*}\right)$ reaches to its maximum when $x_{o}^{*}$ gets close to $x_{m}^{*}$, whereas $Z$ is the portion of kinetic energy that would be dissipated after CL is finally pinned, which can be derived by meeting the condition $E\left(x_{m}^{*}\right)=0$. Because $k$ is related to hysteresis, we assume $k=1+\bar{\phi}$, where $\bar{\phi}=\left(\phi_{L}+\phi_{R}\right) / 2$, the average fraction of the given micro-striated surfaces across the boundary. By solving (4.17)-(4.27), the velocity of the motion, $v$, can be estimated as a function of the position, while the maximum displacement $x_{m}^{*}$ is attained from the experimental results. An example of the evolution of each term involved in (4.25) as a function of $x_{o}^{*}$ is shown in figure 11.

\section{Comparison of experimental results and theory}

First, we compare the vertical oscillation and the truncated models to the experimental results for a wide range of solid fraction difference between striations in figure 7 . For the results related to the smallest solid fraction difference between striations, i.e. the cases closest to a homogeneous striated surface in the absence of contrast in the solid fraction, there is a good agreement between the experimental and the model results are achieved. We note here that the oscillatory behaviour on homogeneous surfaces could further support the theoretical model; however, the static nature of the droplets without the motion in the horizontal direction bring such considerations beyond the purpose of this work. The evolution of the contact radius $r$ and the height of the droplet centroid $(y)$ for both experiments (subscript $e$ ) and models (subscript $m$ ) for five different cases are shown in figure 7 . The best fit for $k_{h}$ in Appendix $\mathrm{C}$ is $k_{h} \approx 1.8$ and the dissipation coefficient, $H$, is $H \approx 0.35 \times 10^{-3} \mathrm{~kg} \mathrm{~s}^{-1}$. For a $9 \mu 1$ water droplet with $m=9 \times 10^{-6} \mathrm{~kg}$ and $\gamma=72 \mathrm{mN} \mathrm{m}^{-1}$, and neglecting the dissipation term, the angular frequency can be estimated as $\omega \approx \sqrt{16 \pi \gamma /(5 m)}$ and the oscillation period, as shown in figure 7(a), is thus $\tau \approx 2 \pi \sqrt{5 \mathrm{~m} /(16 \pi \gamma)}=22 \mathrm{~ms}$, which is in good agreement with our experimental results, at least in the initial stage ( $\tau \approx 20 \mathrm{~ms}$ ). In addition, in the same magnitude, by making use of the Rayleigh equation (Rayleigh 1879), a free droplet gives $\tau \approx 2 \pi \sqrt{m /(8 \pi \gamma)}=14 \mathrm{~ms}$.

Initially, the droplet oscillates with a large amplitude, which suggests that the local, instantaneous variations in Laplace pressure near the CL, caused by distortion of the droplet from spherical, are sufficient to overcome wetting hysteresis and thus prompt outward/inward motion of the CL. Later, as the oscillations become damped as a consequence of energy dissipation, Laplace pressure variations are no longer sufficient to overcome hysteresis. When comparing the theoretical and experimental results of the vertical oscillations of the c.m., it can be seen that the model captures the oscillations quite well. When the c.m. is moving downwards/upwards, the CL is forced to spread/retract and the contact radius increases/decreases. There is a good agreement of the oscillation period between the theoretical and experimental results. In figure $7(f)$, the model captured the dependence of the oscillation period on $\bar{\phi}$, where the period decreases with $\bar{\phi}$. The droplets on surfaces with higher $\bar{\phi}$ experience higher degree of hysteresis, as per the higher solid fraction. Therefore, the CL is pinned more easily during the oscillation, which forces the droplet to oscillate faster until it reaches the equilibrium state, and leads to a lower oscillation period. For the contact radius, there is a rather good qualitative agreement: the droplet spreads and recedes alternately. It is noteworthy that there is a large quantitative discrepancy of the contact radius in figure $7(a, e)$. In the case of figure $7(a)$, the oscillation behaviour is strongest and the droplet overspreads onto the surface, which lead to not only a larger oscillation amplitude of the CL but also a larger value of the final contact radius than expected, because the droplet cannot restore its profile to an equilibrium state owing to 

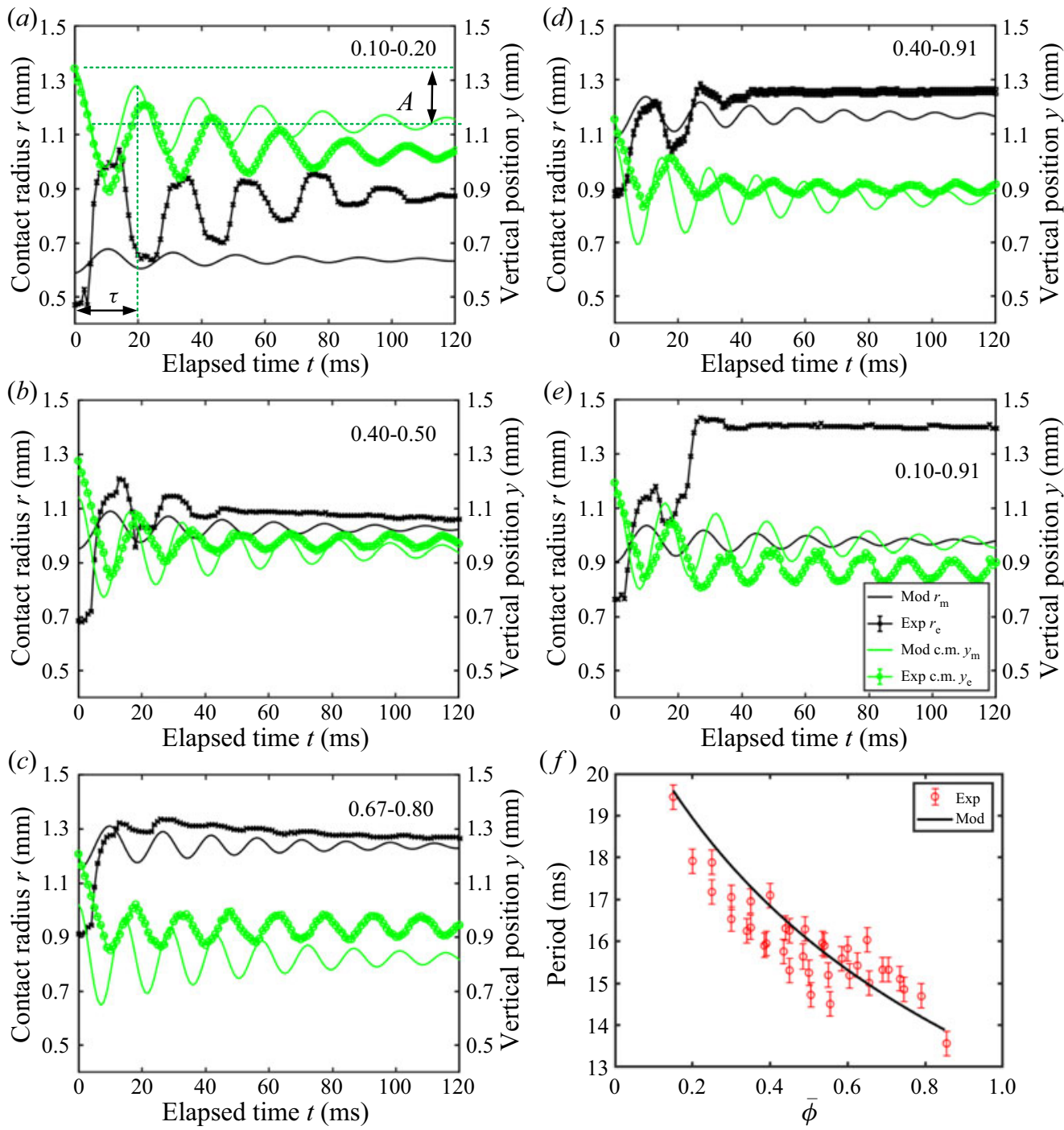

Figure 7. $(a-e)$ Comparison of experimental (subscript $e$ ) and model (subscript $m$ ) results for the vertical oscillation of the c.m., $y(\mathrm{~mm})$, and contact radius, $r(\mathrm{~mm})$, with elapsed time $t(\mathrm{~ms})$ for five different cases. The left and right surface fractions are shown in each graph, e.g. $\left(\phi_{L}=0.10\right)$ and unit $2\left(\phi_{R}=0.20\right)$ in $(a)$. Examples of initial amplitude, $A$, and initial oscillation period, $\tau$, from the model are shown in $(a)$. $(f)$ Dependence of oscillation period on $\bar{\phi}$ for all the experimental cases (red circles) with the result predicted by the model (black line).

hysteresis especially when its CL is receding. In the case of figure 7(e), in the presence of a large $\Delta \phi$, the droplet experiences greater deviations from the spherical shape, i.e.droplet elongation, owing to the preferential spreading onto the right-hand side, which results in the deviation of the contact radius. In the assumption of the model, the distortion of the shapes from equilibrium should be small. An oscillation with a larger amplitude or an elongated shape leads to a bigger deviation between the theoretical and experimental results, mainly in the values of the amplitude, whereas the oscillation of the period are rather well captured. In our earlier work (Zhao et al. 2020), the effect of the amplitude 


\section{H. Zhao, D. Orejon, K. Sefiane and M.E.R. Shanahan}

on the horizontal migration/displacement by releasing the droplet from certain heights was found to increase with increasing the releasing height, i.e. Weber number, within a certain range. This work, however, aims to minimise the influence of the deposition on the reported amplitude, which has proven to be challenging to accurately adjust, so further investigation of the effect of the amplitude on the horizontal migration/displacement by releasing the droplet from certain heights is proposed as the scope for future work.

Just after deposition, the droplet swells in the lower part, owing to a downwards momentum transfer. This increases the local curvature, thus generating a stronger momentary Laplace pressure than for a perfect oblate spheroid, which drives the CL to expand more on the surface than predicted by the model. Thereafter, momentum transfer leads to more liquid in the upper part than in the lower part (figure $2(a)$ at $t=25 \mathrm{~ms}$ ). The reduced volume in the lower part hence reduces the local curvature and a lower Laplace pressure permits the CL to move inwards more than in the case of a perfect prolate spheroid. For the case of a larger $\phi_{R}$, as shown in figure $7(c-e)$, with higher degrees of both wettability and hysteresis, it is more difficult for the CL to shrink, which results in a larger radius. Such perturbations in droplet shape, which are not allowed for in the model, can probably explain, at least to some extent, the reason for the quantitative differences seen between the experimental and model contact radii of the droplet in figure 7 . With the increase of $\bar{\phi}$, the gap between the two results diminishes, because on surfaces with larger $\phi$, the droplet spreads more before it detaches from the needle, and the vertical oscillation becomes weaker as a consequence of the enhanced droplet-surface affinity. Other parameters that contribute to the differences between model and experimental results could lie in the preferable spreading in the striation direction and the gravity effect. Despite these differences, the model describes, in reasonable qualitative and quantitative detail, the vertical oscillatory motion of the c.m. and the lateral alternate leading and trailing motion of the CL.

In figure 8 , the experimental results for droplet velocity, $v$, versus normalised position with respect to the contact radius, $x^{*}=x / r$, are presented, together with the behaviour expected from the model developed previously. Compared with the experimental results for maximum displacement, the best fits for the coefficients, $B^{*}$ and $C$ (in (4.18), (4.19) and (4.26)), are proposed as $B^{*} \approx 18.07 \mathrm{~N} \mathrm{~m}^{-2} \cdot r \Delta \phi / \gamma \bar{\phi}, C \approx 0.13$. In our previous work (Zhao et al. 2020), the average velocity of the droplet, $v$, is a function of $\Delta \phi / \bar{\phi}, \bar{v} \approx$ $\gamma\left(\cos \theta_{Y}+1\right) \Delta \phi /(2 \xi \bar{\phi})$, where $\xi$ is a friction coefficient. Thus, here, $B \propto \bar{v}$.

From figure 8 , good agreement is found between the experimental results and the proposed model. The model describes well how the velocity changes during droplet motion on a structured surface comprising wettability contrast. The droplet c.m. accelerates rapidly at the initial stage, when the driving force is considerably larger than the resistance to motion. Later, when the droplet moves towards the more hydrophilic unit, i.e. a surface with larger $\phi_{R}$, according to (4.16), the length of the boundary covered by the droplet decreases; therefore, the driving force decreases whereas the sum of $f_{L}$ and $f_{R}$ increases. A maximum is reached, followed by deceleration until the drop comes to a halt. The difference between the experimental and model results may arise partially from the assumption that $C$ is considered to be a constant, whereas the different amplitude and period of the oscillations of both $r$ and $y$, the different magnitude of the change in droplet shape caused during deposition and the different droplet surface interactions as a consequence of the different wetting contrast may suggest otherwise. For example, in figure 7, the magnitude of the oscillation as well as the initial contact radius are different for the cases $(a) \phi_{L}=0.10, \phi_{R}=0.20$ and $(b) \phi_{L}=0.40, \phi_{R}=0.50$. 


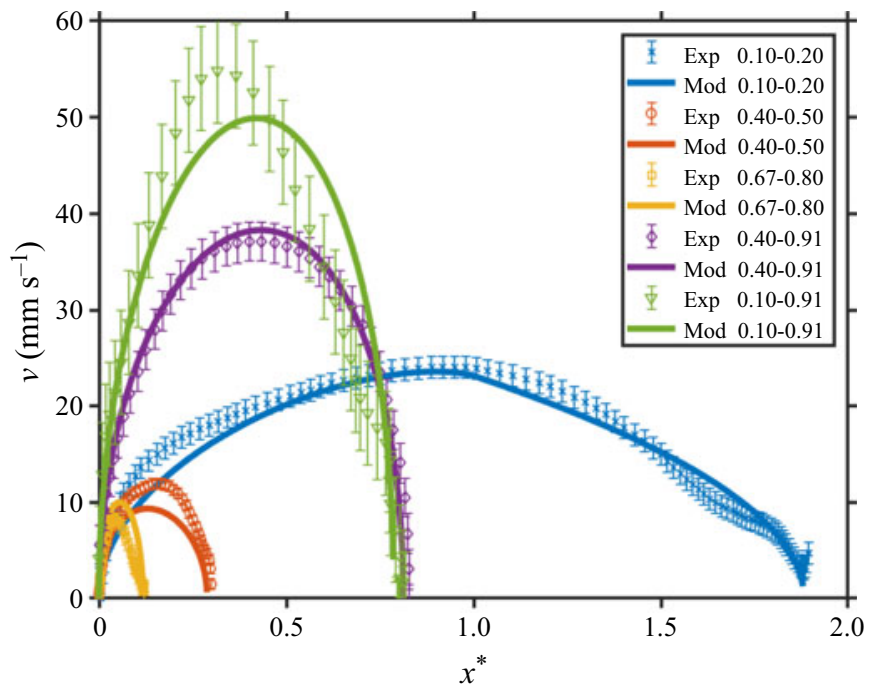

Figure 8. Comparison of the results of droplet velocity, $v\left(\mathrm{~mm} \mathrm{~s}^{-1}\right)$, versus relative position, $x^{*}$, from the experimental results (data points) and model (trend lines) for five different cases. The contrasting boundary between two units is included within the legend where the numbers on the left and right represent the solid fractions for the left and right units, respectively.
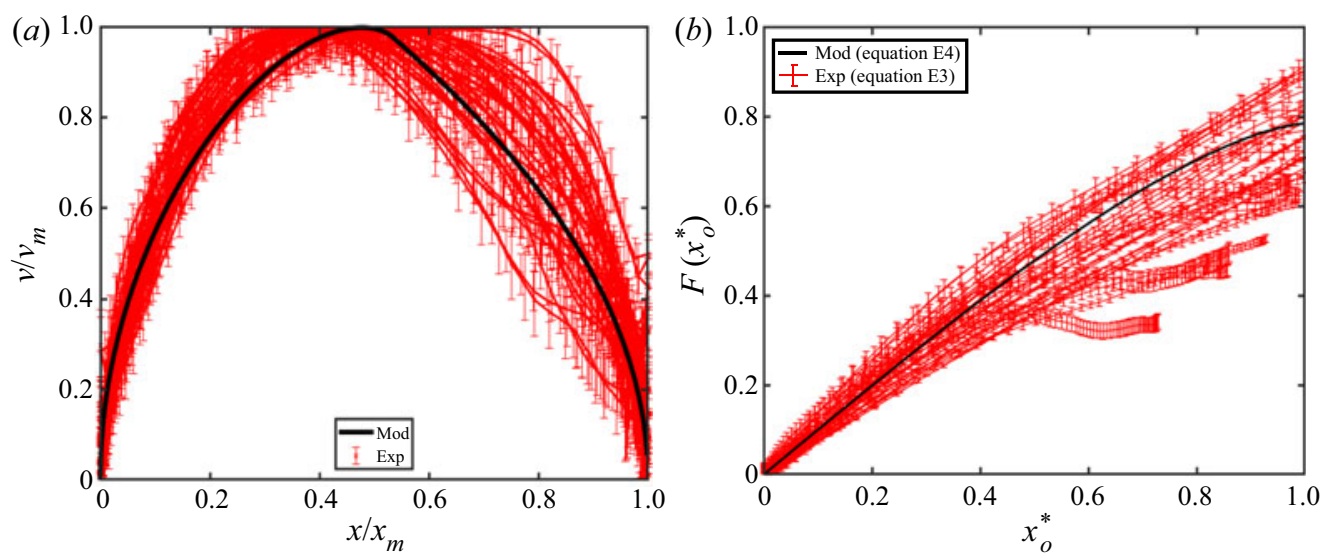

Figure 9. (a) Experimental cases are collapsed by normalising the velocity and the displacement by the maximum velocity, $v_{m}$, and the maximum displacement, $x_{m}$, of each wetting contrast configuration, respectively. The theoretical model of the case $0.10-0.20$ is added for comparison. (b) Comparison of the integral function $F\left(x_{o}^{*}\right)$ derived from experimental values of the dynamic velocity for all wetting contrast configurations and from the theoretical model, where $x_{o}^{*}$ is a given displacement normalised by the droplet contact radius, $r$. The droplet moves across the boundary at $x_{o}^{*}=1$.

We have further collapsed all experimental cases to investigate the dynamic velocity evolution of droplets moving across the wettability contrast by normalising the velocity and the displacement by the maximum velocity $v_{m}$ and maximum displacement $x_{m}$ of each wetting contrast configuration, respectively. The results are presented in figure $9(a)$.

Figure 9 shows that droplets first accelerate upon contacting the surface, then reach the maximum velocity when the driving force and resistance are balanced, and then decelerate until they stop. To understand the evolution of the driving force for all the 


\section{H. Zhao, D. Orejon, K. Sefiane and M.E.R. Shanahan}

different cases regardless of the surface parameters, we present all experimental cases in figure $9(b)$ by re-arranging (4.17)-(4.25) and simplifying some terms (see appendix E). $F\left(x_{o}^{*}\right)$ in figure $9(b)$ is then an indicator of the contribution of the driving force to the kinetic energy as a function of the normalised displacement. Good agreement can be found especially in the early stage. The theoretical value of $F\left(x_{0}^{*}\right)$ at $x_{0}^{*}=1$, where the droplet moves across the boundary, is found to be $F(1)=\pi / 4 \approx 0.79$. For the cases where the droplet can reach $x_{o}^{*}=1$, the experimental value of $F(1)$ varies between 0.6 and 0.9 . The error mainly comes from two sources. One arises from the fact that $W_{D}\left(x_{o}^{*}\right)$ is neglected, then the contribution of the deposition is included into $W_{F_{d}}\left(x_{o}^{*}\right)$, so $F\left(x_{o}^{*}\right)$ is larger than expected. However, because $W_{H}\left(x_{o}^{*}\right)$ is neglected, the energy dissipated after CL pinning is not included, which leads to a smaller $F\left(x_{0}^{*}\right)$. Clear evidence for this is that, for some cases, where the droplet stops before it moves across the boundary totally $\left(x_{o}^{*}=1\right)$, the experimental curve of $F\left(x_{o}^{*}\right)$ deviates greatly from the theoretical curve and stops because the velocity becomes 0 . Therefore, the term $W_{H}\left(x_{o}^{*}\right)$ can no longer be neglected in the cases where the droplet is not able to move fully across the boundary.

\section{Conclusion}

The dynamic behaviour of a droplet moving across the boundary of a wettability contrast micro-structured surface has been experimentally observed and analysed. After being deposited on the boundary, the droplet experiences a vertical damped oscillation and, simultaneously, a horizontal migration. The vertical oscillatory model presented in $\S 4.1$ and the alternating $C L$ slipping model presented in $\S 4.2$ have been developed and coupled to explain the relation between vertical and horizontal motion as well as the behaviour of the CL. In addition, the horizontal migration aspect has been coupled with the vertical oscillation to describe the migration velocity of the droplet in the horizontal migration model presented in $\S 4.3$. The coupling of all three models provides good agreement with the experimental results, which describe the velocity and position of a droplet deposited at a structural contrasting boundary as it migrates towards a denser structured unit. It is noteworthy that not all the cases exhibit a complete droplet migration across the boundary, although both the overshooting and non-overshooting cases have been analysed and captured by the model. Further application of the complete models should enable good prediction of the detailed motion of droplets moving on wetting contrast surfaces or surfaces with wetting contrasts placed in sequence. This is of importance for the optimisation and accurate design of micro-fluidic devices.

Acknowledgements. The authors would like to acknowledge the support of Dr C. Mackenzie-Dover and the Scottish Microelectronics Centre (SMC) for substrate micro-fabrication and surface coating.

Funding. The authors would like to acknowledge the support of the European Space Agency (ESA) through the project Convection and Interfacial Mass Exchange (EVAPORATION) with ESA Contract Number 4000129506/20/NL/PG. The authors also acknowledge the support received from the Engineering and Physical Sciences Research Council (EPSRC) through the grant EP/P005705/1.

Declaration of interests. The authors report no conflict of interest.

Author ORCIDs.

(D) Hongyu Zhao https://orcid.org/0000-0002-1733-976X;

Daniel Orejon https://orcid.org/0000-0003-1037-5036;

Khellil Sefiane https://orcid.org/0000-0003-3300-0210.

916 A $54-18$ 


\section{Droplet motion and oscillation on micro-striated surfaces}

\section{Appendix A. Details on experiment and measurements}

\section{A.1. Surface fabrication}

Four-inch silicon wafers were purchased from Si-Mat (Silicon Materials, Landsberg, Germany). Hexamethyldisilizane (HMDS) was applied to the wafer as an adhesive for the photoresist for $10 \mathrm{~min}$. Silicon wafers were used without a further cleaning procedure as any additional dry or wet cleaning treatment may have induced further deposition of dirt and/or contaminants. Then, the wafers were placed in an SVG 8600 (Silicon Valley Group, USA) track system to dispense and spin coat a SPR 350 photoresist layer with a thickness of $1.2 \mu \mathrm{m}$ onto the surfaces. Following this, the wafers and the photoresist were soft-baked on a heating plate at $90^{\circ} \mathrm{C}$ for $60 \mathrm{~s}$, exposed for $5 \mathrm{~s}$ in a vacuum inside a Karl Suss MA8 mask aligner (Süss, Garching, Germany) and further developed inside the SVG 8600. The substrates were then covered by a pre-designed mask displaying the desired micro-structure patterns and were then exposed to UV light through the mask. After the development process, the exposed resist was washed away, which left the patterned resist on the substrates. The substrates were then subjected to deep reactive-ion etching (deep-RIE, Bosch process) in a surface technology system STS Multiplex ICP (inductively coupled plasma). Etching was carried out for 20 cycles. After etching, the resist was removed from the substrate by acetone, which left the rigid micro-structures with a height of $20 \mu \mathrm{m}$ on the substrates. The surface fabrication was carried out within the Class 10 cleanrooms of the Scottish Microelectronic Centre (SMC). After fabrication, the substrates were sealed in a wafer box and sent to Memsstar Ltd., Scotland for coating. A monolayer of FDTS was applied onto the substrates, which made the surface micro-structures intrinsically hydrophobic.

\section{A.2. Experimental measurements}

CA measurements were conducted on the Drop Shape Analyser 100 (DSA 100, Krüss $\mathrm{GmbH}$, Hamburg, Germany) platform. The apparent CA of the droplet, $\theta_{a p p}$, was measured in both the parallel and orthogonal directions to the micro-striations upon gentle deposition. The $\theta_{a p p}$ measured in the orthogonal direction was found to be dependent on the micro-striation parameters, and the values for different surface units are listed in table 1 . The advancing and receding CAs, $\theta_{a d v}$ and $\theta_{\text {rec }}$, were measured in the orthogonal direction by adding and withdrawing liquid, respectively, which are listed in table 1 . The standard error was estimated from five independent measurements with a system accuracy of $0.3^{\circ}$. We note here that the standard error for all cases was within $3^{\circ}$.

The standard error in the volume of the deposited droplets from five independent measurements and calculations via image processing was estimated as approximately $0.5 \mu \mathrm{l}$. The deviation of the volume resulted from the difficulty to deposit a water droplet onto hydrophobic surfaces with a low solid fraction, i.e. superhydrophobic surfaces. When the volume is small, it is difficult for the water droplet to detach from the needle owing to the low adhesion between the droplet and the hydrophobic surface, and when the volume is large, the surface tension cannot always hold the dosed droplet as a whole, which leaves a small part of the droplet attached to the needle; hence the dosage volume will be less than expected.

Furthermore, the presence of the anisotropic micro-striations induces, to some extent, preferential spreading of the droplet along the striation direction rather than in the orthogonal direction. The anisotropic deviation of the droplet shape was noted by the use 


\section{H. Zhao, D. Orejon, K. Sefiane and M.E.R. Shanahan}

of a top view camera, and the difference of droplet radii measured along the two directions was estimated to be within about $7 \%$.

\section{Appendix B. Derivation details on vertical oscillation of a spherical droplet}

As stated in (4.1), the radius, $a_{s}$, of the equivalent spherical droplet is given by

$$
a_{s}=\sqrt[3]{\frac{3 V_{s}}{4 \pi}},
$$

where $V_{s}$ is the volume of the spheroid. The spherical droplet changes its shape from prolate $(\varepsilon>0)$ to oblate $(\varepsilon<0)$ with an alternate positive to negative perturbation in the vertical position of c.m., $\varepsilon$. Therefore, the polar radius of the prolate (subscript $p$ ) or oblate (subscript $o$ ) is $a_{p, o}=a_{s}+\varepsilon$. The equatorial radius of the spheroid, $b_{p}$ or $b_{o}$ is given by

$$
b_{p, o}=\sqrt{\frac{3 V_{s}}{4 \pi\left(a_{s}+\varepsilon\right)}}=\sqrt{\frac{a_{s}^{3}}{a_{s}+\varepsilon}} .
$$

To evaluate the free energy of the droplet associated with its surface, the surface area of the equivalent spheroid, $S_{p, o}$, is required. The surface area for a prolate spheroid, $S_{p}$, is given by (Weisstein 2003c)

$$
S_{p}=2 \pi b_{p}^{2}\left(1+\frac{a_{p}}{b_{p} e_{p}} \sin ^{-1} e_{p}\right),
$$

where the eccentricity $e_{p}=\sqrt{1-\left(b_{p} / a_{p}\right)^{2}}=\sqrt{1-\left(a_{s} /\left(a_{s}+\varepsilon\right)\right)^{3}}$. Using the Maclaurin series expansion (Weisstein 2003a), $S_{p}$ can be written as

$$
S_{p}=2 \pi \frac{a_{s}^{3}}{a_{s}+\varepsilon}\left(1+\sqrt{\frac{\left(a_{s}+\varepsilon\right)^{3}}{a_{s}^{3} e_{p}^{2}}} \sin ^{-1} e_{p}\right) \approx 4 \pi a_{s}^{2}\left(1+\frac{2 \varepsilon^{2}}{5 a_{s}^{2}}\right) .
$$

For an oblate spheroid surface, $S_{o}$ can be written as (Weisstein 2003b)

$$
S_{o}=2 \pi b_{o}^{2}+\pi \frac{a_{o}^{2}}{e_{o}} \ln \frac{1+e_{o}}{1-e_{o}},
$$

where the eccentricity $e_{o}=\sqrt{1-\left(a_{o} / b_{o}\right)^{2}}=\sqrt{1-\left(\left(a_{s}+\varepsilon\right) / a_{S}\right)^{3}}$. As previously, $S_{o}$ can be written as

$$
S_{o} \approx 4 \pi a_{s}^{2}\left(1+\frac{2 \varepsilon^{2}}{5 a_{s}^{2}}\right) .
$$

Thus, to a first approximation, the surface areas of prolate and oblate spheroids, when only slightly deformed, are the same, $S_{p}=S_{o}$, and given by either (B4) or B6. Neglecting gravity, as is reasonable in the present context, the potential energy, $P$, of a perturbed drop is driven by surface tension and thus given by

$$
P \approx 4 \pi \gamma a_{s}^{2}\left(1+\frac{2 \varepsilon^{2}}{5 a_{s}^{2}}\right),
$$

where $\gamma$ is the liquid-vapour surface tension of the droplet. The internal kinetic energy of the droplet, $K$, can be defined as $K \approx 1 / 2 m \dot{\varepsilon}^{2}$, where $m$ is the droplet mass. A more 
complete analysis allowing for shape change contributions to $K$ leads to a pre-factor of $27 / 40$ instead of $1 / 2$, but this leads to undue complication and unwarranted precision (for more details on a more complete analysis, see appendix D). Whereas the internal viscous dissipation, $D$, as $D \approx 1 / 2 H \dot{\varepsilon}^{2}$, where $H$ is a dissipation coefficient, may be related to liquid viscosity and/or to the surface structure; nonetheless, for the sake of simplicity, the model assumes a constant value. Further analysis demonstrated that the variability of $H$ as a function of $\bar{\phi}$ was found to have negligible impact (i.e. a low contribution of one or two orders of magnitude) on predicting the period of oscillations. In light of the agreement obtained between the model and the experiment, this seems to be a reasonable assumption while keeping the model as simple as possible. We construct the Lagrangian function, $L$ :

$$
L=K-P=\frac{1}{2} m \dot{\varepsilon}^{2}-4 \pi \gamma a_{s}^{2}\left(1+\frac{2 \varepsilon^{2}}{5 a_{s}^{2}}\right) .
$$

The corresponding Lagrange equation of the first kind with respect to the dynamic deviation from sphericity, $\dot{\varepsilon}$, is

$$
\frac{\mathrm{d}}{\mathrm{d} t}\left(\frac{\partial L}{\partial \dot{\varepsilon}}\right)-\frac{\partial L}{\partial \varepsilon}+\frac{\partial D}{\partial \dot{\varepsilon}}=0
$$

From (B8) and (B9), we derive

$$
m \ddot{\varepsilon}+H \dot{\varepsilon}+\frac{16 \pi \gamma \varepsilon}{5}=0
$$

Solving (B10), we obtain the time-dependent deviation of the polar radius of the spheroid, $\varepsilon(t)$ :

$$
\varepsilon(t)=A \exp \left(-\frac{H t}{2 m}\right) \cos \left(\sqrt{\frac{16 \pi \gamma}{5 m}-\frac{H^{2}}{4 m^{2}}} \cdot t+\beta\right) .
$$

And the time-dependent polar radius of the spheroid, $a(t)$, is

$$
a(t)=a_{s}+\varepsilon(t)=a_{s}+A \exp \left(-\frac{H t}{2 m}\right) \cos \left(\sqrt{\frac{16 \pi \gamma}{5 m}-\frac{H^{2}}{4 m^{2}}} \cdot t+\beta\right) .
$$

\section{Appendix C. Derivation details on vertical oscillation of a truncated spherical droplet}

The volume of a truncated sphere, $V_{t s}$, is given by

$$
V_{t s}=\int_{-y_{t s}}^{a_{t s}} \pi x^{2} \mathrm{~d} y=\frac{\pi}{3}\left(2 a_{t s}^{3}+3 a_{t s}^{2} y_{t s}-y_{t s}^{3}\right)=\frac{\pi}{3} a_{t s}^{3}\left(1-\cos \theta^{*}\right)^{2}\left(2+\cos \theta^{*}\right),
$$

where $\theta^{*}$ (equivalent to the apparent CA of the droplet, but here is simply a geometrical concept) is the interior angle subtended between the tangent to the spheroid and the truncation plane at their intersection and $y_{t s}=-a_{t s} \cos \theta^{*}$. The base radius of a truncated sphere (contact radius of the droplet), $r_{t s}$, is $r_{t s}=a_{t s} \sin \theta^{*}$. 


\section{H. Zhao, D. Orejon, K. Sefiane and M.E.R. Shanahan}

For a truncated prolate or oblate spheroid shown in figure 4 (left) and (right), respectively, the volume, $V$, is given by

$$
V=\int_{-y_{p, o}}^{a} \pi b^{2}\left(1-y_{p, o}^{2} / a^{2}\right) \mathrm{d} y=\frac{\pi}{3} b^{2}\left(2 a+3 y_{p, o}-\frac{y_{p, o}^{3}}{a^{2}}\right) .
$$

With the constant volume condition:

$$
b^{2}\left(2 a+3 y_{p, o}-\frac{y_{p, o}^{3}}{a^{2}}\right)=2 a_{t s}^{3}+3 a_{t s}^{2} y_{t s}-y_{t s}^{3} .
$$

By comparing full and truncated spheroids (figures 3 and 4), therefore taking $a_{t s}=a_{s}$, $b=b_{p, o}=\sqrt{a_{s}^{3} /\left(a_{s}+\varepsilon\right)}$, we have $a=a_{t s}+\varepsilon$ and (C3) becomes

$$
a_{t s}^{3}\left[2+\frac{3 y_{p, o}}{a_{t s}+\varepsilon}-\frac{y_{p, o}^{3}}{\left(a_{t s}+\varepsilon\right)^{3}}\right]=a_{t s}^{3}\left(2+\frac{3 y_{t s}}{a_{t s}}-\frac{y_{t s}^{3}}{a_{t s}^{3}}\right) .
$$

The real solution of this equation is

$$
y_{p, o}=\frac{y_{t s}\left(a_{t s}+\varepsilon\right)}{a_{t s}},
$$

and the base radius, $r$, can be written as

$$
r=b \sqrt{\left(1-\frac{y^{2}}{a^{2}}\right)}=b \sqrt{\left(1-\frac{y_{t s}^{2}}{a_{t s}^{2}}\right)} \approx a_{t s} \sin \theta^{*}\left(1-\frac{\varepsilon}{2 a_{t s}}\right)=r_{t s}\left(1-\frac{\varepsilon}{2 a_{t s}}\right) .
$$

When the droplet contacts the solid with a finite contact area, the CL needs to overcome hysteresis before it can move. Compared with the ideal cases without hysteresis, the existence of hysteresis in the realistic case distorts the droplet shape from the spheroid shape, i.e. part of the kinetic energy is 'stored' temporally, which should be a function of the surface area as well as the degree of hysteresis. Accounting for this part of the energy, we introduce another term, $-h P=-4 \pi \gamma a_{s}^{2}\left(1+2 \varepsilon^{2} / 5 a_{s}^{2}\right) h$, in (B8):

$$
L=K-P=\frac{1}{2} m \dot{\varepsilon}^{2}-4 \pi \gamma a_{s}^{2}\left(1+\frac{2 \varepsilon^{2}}{5 a_{s}^{2}}\right)(1+h),
$$

where $h$ is the coefficient of the stored energy by surface distortion via hysteresis. According to (B12) and (C5), the vertical position of $y(t)$ is

$$
y(t)=y_{t s}+A^{\prime} \exp \left(-\frac{H t}{2 m}\right) \cos \left(\sqrt{\frac{16 \pi \gamma}{5 m}(1+h)-\frac{H^{2}}{4 m^{2}}} \cdot t+\beta\right),
$$

where $A^{\prime}=A y_{t s} / a_{t s}$ and the coefficient, $h$, should be a function of $\bar{\phi}$, which presents the degree of hysteresis, so we assume the expression for $h$ is $h=k_{h} \bar{\phi} \gamma$, where $\bar{\phi}=\left(\phi_{L}+\right.$ $\left.\phi_{R}\right) / 2$ and $k_{h}$ is set to be a constant for the sake of simplicity of the model. It is noteworthy that the surface distortion term, $-h P$, only exists when the CL is pinned, and it is released as kinetic energy as soon as the CL moves. From figure 2(c), we know that the CL is pinned 


\section{Droplet motion and oscillation on micro-striated surfaces}

when $\varepsilon^{2}$ is at its maxima and moves when $\varepsilon^{2}$ is small; therefore, for the simplicity of the model, we assume that the distortion term induced by hysteresis continuously changes with $\varepsilon^{2}$. Equation (C8) is applied to the derivation of the results in figure 7. It is noteworthy that $y(t)$ is the vertical position of the centre of the spheroid above the surface, and the geometric centroid, i.e. the c.m. of the droplet, is above $y(t)$ by $3 / 4 R\left(1+\cos \theta^{*}\right)^{2} /(2+$ $\cos \theta^{*}$ ) (Harris \& Stöcker 1998). This has been taken into account in $y_{m}$ in figure 7.

\section{Appendix D. Derivation of kinetic energy of the droplet}

For the whole spheroid, normalising $\varepsilon$ by $a_{s}$ so $\varepsilon^{*}=\varepsilon / a_{S}$, we have $a_{p, o}=a_{s}+\varepsilon=a_{s}(1+$ $\left.\varepsilon^{*}\right)$, and (B2) becomes

$$
b_{p, o}=\sqrt{\frac{a_{s}^{3}}{a_{s}+\varepsilon}}=a_{s}\left(1+\varepsilon^{*}\right)^{-1 / 2} .
$$

Taking the oblate spheroid case in figure 10 as an example $(\varepsilon<0)$, where $r_{o s}$ represents the radius of the horizontal cross-section circle with a vertical distance from the origin, $y$, the distance between a point on the horizontal cross-section circle to the centre of the certain circle, $r(y)=\sqrt{x^{2}+z^{2}}$, obeys $0 \leq r(y) \leq r_{o s}(y)$. Using the equation for a vertically axisymmetric ellipsoid, $\left(x^{2}+z^{2}\right) / b^{2}+y^{2} / a^{2}=1$, we have

$$
\begin{aligned}
& \frac{r_{o s}^{2}(y)}{a_{s}^{2}}\left(1+\varepsilon^{*}\right)+\frac{y^{2}}{a_{s}^{2}}\left(1+\varepsilon^{*}\right)^{-2}=1, \\
& r_{o s}^{2}\left(y^{*}\right)=a_{s}^{2}\left[\frac{1}{1+\varepsilon^{*}}+\frac{y^{* 2}}{\left(1+\varepsilon^{*}\right)^{3}}\right],
\end{aligned}
$$

where $y^{*}=y / a_{s}$. During the vertical oscillation, by homothety, we assume the local vertical velocity inside the spheroid, $v_{v}$, increases linearly with height and with the boundary conditions $v_{v}=0$, where $y=-a$, i.e. the spheroid contacts the wall, and $v_{v}=\dot{\varepsilon}$, where $y=0$, i.e. the c.m. of the droplet has a vertical velocity $\dot{\varepsilon}$. Then the vertical velocity at $(y, r)$ within the oblate spheroid is $v_{v}=\dot{\varepsilon}(1+y / a)=a_{s} \dot{\varepsilon}^{*}\left[1+y^{*} /\left(1+\varepsilon^{*}\right)\right]$, where $\dot{\varepsilon}^{*}=\dot{\varepsilon} / a_{s}$.

Assume a droplet with a density of $\rho$ has the same shape as the oblate spheroid, then a disc of thickness $a_{s} \delta y^{*}$ from the droplet has a vertical energy, $K_{v}=1 / 2 \delta m v_{v}^{2}$, of

$$
K_{v}\left(y^{*}\right)=\pi \rho r_{o s}^{2} a_{s} \delta y^{*} \frac{1}{2} v_{v}^{2}=\frac{\pi}{2} \rho a_{s}^{5} \delta y^{*} \dot{\varepsilon}^{* 2}\left[\frac{1}{1+\varepsilon^{*}}+\frac{y^{* 2}}{\left(1+\varepsilon^{*}\right)^{3}}\right]\left(1+\frac{y^{* 2}}{1+\varepsilon^{*}}\right)^{2} .
$$

Taking $Y=y^{*} /\left(1+\varepsilon^{*}\right)=y / a$ and $\delta Y=\delta y /\left[a_{s}\left(1+\varepsilon^{*}\right)\right]$, (D4) becomes

$$
K_{v}(Y)=\frac{\pi}{2} \rho a_{s}^{5} \delta Y \dot{\varepsilon}^{* 2}\left(1-Y^{2}\right)(1+Y)^{2} .
$$

Because $-1<Y<1$, by integrating $K_{v}(Y)$ from -1 to 1 , the total vertical internal kinetic energy, $K_{t v}$, is derived as

$$
K_{t v}=\frac{\pi}{2} \rho a_{s}^{5} \dot{\varepsilon}^{* 2} \int_{-1}^{1}\left(1-Y^{2}\right)(1+Y)^{2} \mathrm{~d} Y=\frac{4}{5} \pi \rho a_{s}^{5} \dot{\varepsilon}^{* 2}=\frac{3}{5} m \dot{\varepsilon}^{2},
$$

where $m$ is the whole mass of the droplet. 


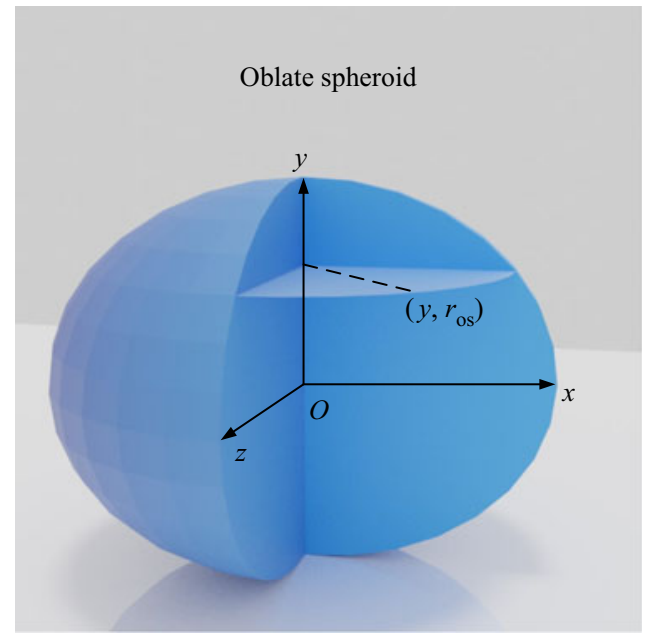

Figure 10. Schematic diagram of the whole oblate spheroid. A random point on the surface is shown in the figure with the coordinates $\left(y, r_{o s}\right)$. Here $r_{o s}=\sqrt{x^{2}+z^{2}}$, where $x$ and $z$ are the coordinates along the $x$ and $z$ axes.

Furthermore, there exists a horizontal component of internal $K$. By homothety, we assume a linear local horizontal velocity inside the spheroid along the cross-section radius direction, $v_{h}=\dot{r}_{O S} r / r_{O S}$, where $\dot{r}_{O S}$ is the horizontal velocity at $r=r_{O S}$, i.e. on the cross-section circle at a given $y$, which can be derived from (D3):

$$
\dot{r}_{o s}=\frac{-1+3 y^{* 2} /\left(1+\varepsilon^{*}\right)^{2}}{2\left(1+\varepsilon^{*}\right)^{2} r_{o s}} a_{s}^{2} \dot{\varepsilon}^{*}
$$

Then a disc of thickness $\delta y=a_{s} \delta y^{*}$ from the droplet has a horizontal kinetic energy, $K_{h}\left(y^{*}\right)$, of

$$
K_{h}\left(y^{*}\right)=\int_{0}^{r_{o s}} \frac{1}{2} v_{h}^{2} 2 \pi r \rho \mathrm{d} r \delta y=\frac{\pi \rho \dot{r}_{o s}^{2}}{r_{o s}^{2}} \int_{0}^{r_{o s}} r^{3} \mathrm{~d} r=\frac{\pi}{4} \rho r_{o s}^{2} \dot{r}_{o s}{ }^{2} a_{S} \delta y^{*} .
$$

With (D7), $K_{h}(Y)$ is

$$
K_{h}(Y)=\frac{\pi}{4} \rho r_{o s}^{2} \dot{r}_{o s}{ }^{2} a_{s} \delta y^{*}=\frac{\pi}{16\left(1+\varepsilon^{*}\right)^{3}} \rho a_{s}^{5} \dot{\varepsilon}^{* 2}\left(-1+3 Y^{2}\right)^{2} \delta Y .
$$

Taking that $\varepsilon^{*} \ll 1$, the total horizontal kinetic energy $K_{t h}$ is

$$
K_{t h}=\frac{\pi}{16} \rho a_{s}^{5} \dot{\varepsilon}^{* 2} \int_{-1}^{1}\left(-1+3 Y^{2}\right)^{2} \mathrm{~d} Y=\frac{1}{10} \pi \rho a_{s}^{5} \dot{\varepsilon}^{* 2}=\frac{3}{40} m \dot{\varepsilon}^{2},
$$

then the total internal kinetic energy, $K$, is

$$
K=K_{t v}+K_{t h}=\frac{27}{40} m \dot{\varepsilon}^{2} .
$$

The derivation of $K$ is the same for a prolate spheroid with $\varepsilon>0$. 


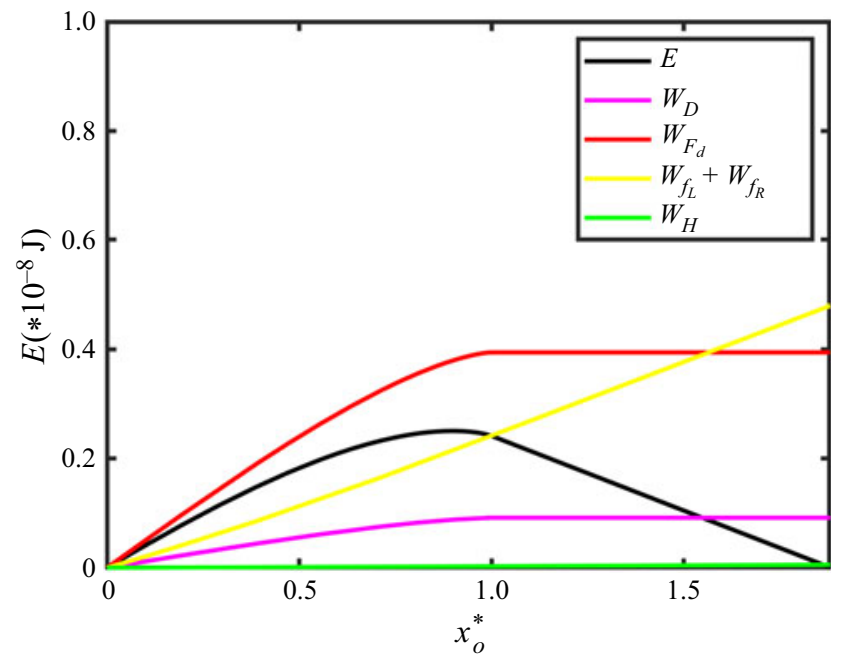

Figure 11. The works done by different forces in the case with $\phi_{L}=0.10$ and $\phi_{R}=0.20$, from 0 to a given displacement, $x_{o}^{*}=x_{o} / r$, where $r$ is the contact radius of the droplet. The evolution of each term in (E1) is presented.

\section{Appendix E. Rearrangement and simplification of the horizontal migration model}

To determine the evolution of the driving force for all the different cases, we re-arrange (4.17)-(4.25) and make some simplifications. First, the kinetic energy (4.25) for $x_{o}^{*}<1$ is

$$
E\left(x_{o}^{*}\right)=W_{D}\left(x_{o}^{*}\right)+W_{F_{d}}\left(x_{o}^{*}\right)-W_{f_{L}}\left(x_{o}^{*}\right)-W_{f_{R}}\left(x_{o}^{*}\right)-W_{H}\left(x_{o}^{*}\right)=\frac{1}{2} m v^{2},
$$

an example of the contribution of each term in (E1) for the case, $\phi_{L}=0.10$ and $\phi_{R}=0.20$, is shown in figure 11. If we now assume that the only driving force is $F_{d}$ and neglect the term $W_{D}\left(x_{o}^{*}\right)$ (in most of the cases, $W_{F_{d}}\left(x_{o}^{*}\right) / W_{D}\left(x_{o}^{*}\right)>4$ ), and because $W_{H}\left(x_{o}^{*}\right)$ plays its role mainly near the end of the motion, we arrive at the following simplified energy equation:

$$
W_{F_{d}}\left(x_{o}^{*}\right) \approx \frac{1}{2} m v^{2}+W_{f_{L}}\left(x_{o}^{*}\right)+W_{f_{R}}\left(x_{o}^{*}\right) .
$$

By making use of (4.7), (4.17)-(4.19) and (4.21)-(4.23), the integral function $F\left(x_{o}^{*}\right)$ derived from experimental values of the dynamic velocity can be expressed as

$$
F\left(x_{o}^{*}\right)=\frac{W_{F_{d}}\left(x_{o}^{*}\right)}{2 \pi \gamma r^{2}\left(1+\cos \theta_{Y}\right) \Delta \phi} \approx \frac{\frac{1}{2} m v^{2}+W_{f_{L}}\left(x_{o}^{*}\right)+W_{f_{R}}\left(x_{o}^{*}\right)}{2 \pi \gamma r^{2}\left(1+\cos \theta_{Y}\right) \Delta \phi} .
$$

We also know that the theoretical expression for $F\left(x_{o}^{*}\right)$ from (4.21) is

$$
F\left(x_{o}^{*}\right)=\int_{0}^{x_{o}^{*}} \sqrt{1-\left(x^{*}\right)^{2}} \mathrm{~d} x^{*} .
$$

It is now possible to collapse all experimental data using (E3) and compare the results with the theoretical (E4). It is noteworthy that the only fitting parameter in (E3) is $B^{*} \approx 18.07 \mathrm{~N} \mathrm{~m}^{-2} \cdot r \Delta \phi / \gamma \bar{\phi}$. We cannot further simplify the expression because of the complicated forms of $W_{f_{L}}\left(x_{o}^{*}\right)$ and $W_{f_{R}}\left(x_{o}^{*}\right)$. Experimental and model results using (E3) and (E4) are then compared in figure $9(b)$. Because $W_{F_{d}}\left(x_{o}^{*}\right)=2 \pi \gamma r^{2}\left(1+\cos \theta_{Y}\right) \Delta \phi F\left(x_{o}^{*}\right)$, $F\left(x_{o}^{*}\right)$ acts as an indicator of the contribution of the driving force to the kinetic energy as a function of normalised displacement. 


\section{H. Zhao, D. Orejon, K. Sefiane and M.E.R. Shanahan}

\section{REFERENCES}

Bartolo, D., Josserand, C. \& Bonn, D. 2005 Retraction dynamics of aquous drops upon impact on nonwetting surfaces. J. Fluid Mech. 545, 329-338.

Becker, E., Hiller, W. \& Kowalewski, T. 1991 Experimental and theoretical investigation of large-amplitude oscillations of liquid droplets. J. Fluid Mech. 231, 189-210.

Blossey, R. 2003 Self-cleaning surfaces-virtual realities. Nat. Mater. 2 (5), 301-306.

Brunet, P., Eggers, J. \& DeEgan, R. 2007 Vibration-induced climbing of drops. Phys. Rev. Lett. 99 (14), 144501.

CAssie, A. \& BAXter, S. 1944 Wettability of porous surfaces. Trans. Far. Soc. 40, 546-551.

Chaudhury, M.K. \& Whitesides, G.M. 1992 How to make water run uphill. Science 256 (5063), 1539-1541.

Chevy, F., Chepelianskit, A., QuÉRÉ, D. \& RAPHAËL, E. 2012 Liquid hertz contact: softness of weakly deformed drops on non-wetting substrates. Europhys. Lett. 100 (5), 54002.

Chiou, P.Y., Chang, Z. \& Wu, M.C. 2008 Droplet manipulation with light on optoelectrowetting device. J. Microelectromech. Syst. 17 (1), 133-138.

Courty, S., Lagubeau, G. \& Tixier, T. 2006 Oscillating droplets by decomposition on the spherical harmonics basis. Phys. Rev. E 73 (4), 045301.

Daniel, S., Chaudhury, M.K. \& Chen, J.C. 2001 Fast drop movements resulting from the phase change on a gradient surface. Science 291 (5504), 633-636.

De Angelis, F., Gentile, F., Mecarini, F., Das, G., Moretti, M., Candeloro, P., Coluccio, M., Cojoc, G., Accardo, A. \& Liberale, C., et al.2011 Breaking the diffusion limit with super-hydrophobic delivery of molecules to plasmonic nanofocusing sers structures. Nat. Photonics 5 (11), 682-687.

Dong, Y., Holmes, H.R. \& BÖHRINGER, K.F. 2017 Converting vertical vibration of anisotropic ratchet conveyors into horizontal droplet motion. Langmuir 33 (40), 10745-10752.

Duncombe, T.A., Erdem, E.Y., Shastry, A., Baskaran, R. \& Böhringer, K.F. 2012 Controlling liquid drops with texture ratchets. Adv. Mater. 24 (12), 1545-1550.

Gordillo, J.M., Riboux, G. \& Quintero, E.S. 2019 A theory on the spreading of impacting droplets. J. Fluid Mech. 866, 298-315.

HARRIS, J.W. \& STÖCKER, H. 1998 Handbook of Mathematics and Computational Science. Springer Science \& Business Media.

HE, B. \& LEE, J. 2003 Dynamic wettability switching by surface roughness effect. In The Sixteenth Annual International Conference on Micro Electro Mechanical Systems, 2003. MEMS-03 Kyoto. IEEE, pp. 120-123. IEEE.

JunG, Y.C. \& BHUSHAN, B. 2008 Dynamic effects of bouncing water droplets on superhydrophobic surfaces. Langmuir 24 (12), 6262-6269.

Kim, J., Moon, M.-W. \& Kim, H.-Y. 2016 Dynamics of hemiwicking. J. Fluid Mech. 800, 57-71.

Kita, Y., Dover, C.M., Askounis, A., TAkata, Y. \& Sefiane, K. 2018 Drop mobility on superhydrophobic microstructured surfaces with wettability contrasts. Soft Matt. 14 (46), 9418-9424.

LAERMER, F. \& SCHILP, A. 1996 Method for anisotropic plasma etching of substrates. US Patent 5, $498,312$.

Launay, G., Sadullah, M.S., Mchale, G., Ledesma-Aguilar, R., KusumaAtmaja, H. \& Wells, G.G. 2020 Self-propelled droplet transport on shaped-liquid surfaces. Sci. Rep. 10 (1), 1-8.

Lyubimov, D.V., Lyubimova, T.P. \& ShklyaeV, S.V. 2006 Behavior of a drop on an oscillating solid plate. Phys. Fluids 18 (1), 012101.

Macner, A.M., DAniel, S. \& SteEn, P.H. 2014 Condensation on surface energy gradient shifts drop size distribution toward small drops. Langmuir 30 (7), 1788-1798.

Moradi, N., VARniK, F. \& STEINBACH, I. 2010 Roughness-gradient-induced spontaneous motion of droplets on hydrophobic surfaces: A lattice Boltzmann study. Europhys. Lett. 89 (2), 26006.

Morrison, C.A., LeavitT, R. \& Wortman, D. 1981 The extended rayleigh theory of the oscillation of liquid droplets. J. Fluid Mech. 104, 295-309.

ÖNER, D. \& MCCARThY, T.J. 2000 Ultrahydrophobic surfaces, effects of topography length scales on wettability. Langmuir 16 (20), 7777-7782.

Noblin, X., Kofman, R. \& Celestini, F. 2009 Ratchetlike motion of a shaken drop. Phys. Rev. Lett. $102(19), 194504$.

RAYLEIGH, LORD 1879 On the capillary phenomena of jets. Proc. R. Soc. Lond. 29 (196-199), 71-97.

REYSsat, M., PARDo, F. \& QuÉRÉ, D. 2009 Drops onto gradients of texture. Europhys. Lett. 87 (3), 36003.

Shastry, A., CASE, M.J. \& BÖHRINGER, K. 2005 Engineering surface roughness to manipulate droplets in microfluidic systems. In 18th IEEE International Conference on Micro Electro Mechanical Systems, 2005. MEMS 2005, pp. 694-697. IEEE. 


\section{Droplet motion and oscillation on micro-striated surfaces}

Shastry, A., CASe, M.J. \& BÖHRinger, K.F. 2006 Directing droplets using microstructured surfaces. Langmuir 22 (14), 6161-6167.

SHASTRY, A., TAYLOR, D. \& BOHRINGER, K.F. 2007 Micro-structured surface ratchets for droplet transport. In TRANSDUCERS 2007-2007 International Solid-State Sensors, Actuators and Microsystems Conference, pp. 1353-1356. IEEE.

Soto, Á.M., Maddalena, T., Fraters, A., Van Der Meer, D. \& Lohse, D. 2018 Coalescence of diffusively growing gas bubbles. J. Fluid Mech. 846, 143-165.

SRinivasan, V., Pamula, V.K. \& FAir, R.B. 2004 An integrated digital microfluidic lab-on-a-chip for clinical diagnostics on human physiological fluids. Lab Chip 4 (4), 310-315.

Velev, O.D., Prevo, B.G. \& BHATT, K.H. 2003 On-chip manipulation of free droplets. Nature 426 (6966), $515-516$.

WeissteIN, E.W. 2003 Maclaurin series. https://mathworld.wolfram.com/.

WEISSTEIN, E.W. 2003b Oblate spheroid. https://mathworld.wolfram.com/.

WeISSTEIN, E.W. 2003c Prolate spheroid. https://mathworld.wolfram.com/.

WENZEL, R.N. 1936 Resistance of solid surfaces to wetting by water. Ind. Eng. Chem. 28 (8), 988-994.

Wildeman, S., Visser, C.W., Sun, C. \& Lohse, D. 2016 On the spreading of impacting drops. J. Fluid Mech. 805, 636-655.

Wixforth, A., Strobl, C., Gauer, C., Toegl, A., Scriba, J. \& Guttenberg, Z.V. 2004 Acoustic manipulation of small droplets. Anal. Bioanal. Chem. 379 (7-8), 982-991.

XU, W. \& CHOI, C.-H. 2012 From sticky to slippery droplets: dynamics of contact line depinning on superhydrophobic surfaces. Phys. Rev. Lett. 109 (2), 024504.

YAng, J.-T., Chen, J.C., HuAng, K.-J. \& YeH, J.A. 2006 Droplet manipulation on a hydrophobic textured surface with roughened patterns. J. Microelectromech. Syst. 15 (3), 697-707.

Yeh, S.I., FAng, W.F., SheEN, H.J. \& YANG, J.T. 2013 Droplets coalescence and mixing with identical and distinct surface tension on a wettability gradient surface. Microfluid. Nanofluidics 14 (5), 785-795.

Yoshimitsu, Z., Nakajima, A., Watanabe, T. \& Hashimoto, K. 2002 Effects of surface structure on the hydrophobicity and sliding behavior of water droplets. Langmuir 18 (15), 5818-5822.

Young, T. 1805 III. An essay on the cohesion of fluids. Phil. Trans. R. S. Lond. 95, 65-87.

Zhao, H., Orejon, D., Mackenzie-Dover, C., Valluri, P., Shanahan, M.E.R. \& Sefiane, K. 2020 Droplet motion on contrasting striated surfaces. Appl. Phys. Lett. 116 (25), 251604.

Zheng, Y., Bai, H., Huang, Z., Tian, X., Nie, F.-Q., Zhao, Y., Zhai, J. \& Jiang, L. 2010 Directional water collection on wetted spider silk. Nature 463 (7281), 640-643. 